\title{
Quantum tunneling in two-dimensional van der Waals heterostructures and devices
}

\author{
Sidi Fan, Rui Cao, Lude Wang, Shan Gao, Yupeng Zhang, Xiang Yu ${ }^{*}$ and Han Zhang*
}

\begin{abstract}
Quantum tunneling with band-structure engineering has been feasibly developed for many applications in electrical, optoelectrical, and magnetic devices. It relies on layer-by-layer design and fabrication, which is an interdisciplinary research field covering material science and technology. Ever since the discovery of two-dimensional (2D) layered materials, tunneling devices based on 2D van der Waals (vdW) heterostructures have been extensively studied as potential next-generation devices. $2 \mathrm{D}$ materials are thin at the atomic scale and extremely flat without surface dangling bonds. Because of these unique characteristics, $2 \mathrm{D}$ vdW heterostructures offer superior tunneling performance that reaches the benchmark of traditional Si technology and possess additional ability to scale down device size. Here, we comprehensively review quantum tunneling in $2 \mathrm{D}$ vdW heterostructures, in addition to their unique mechanisms and applications. Moreover, we analyze the possibilities and challenges currently faced by $2 \mathrm{D}$ tunneling devices and provide a perspective on their exploitation for advanced future applications. The investigation of technology- and performancecontrol of $2 \mathrm{D}$ tunneling devices is at their beginning stages; however, these devices should emerge as competitive candidates for realizing low-power supply, fast-speed capability, and high-frequency operating devices.
\end{abstract}

Keywords: quantum tunneling, 2D materials, van der Waals heterostructures, $2 \mathrm{D}$ quantum devices

\section{INTRODUCTION}

Quantum tunneling is a quantum mechanical phenomenon in which a subatomic particle (such as an electron, photon, or phonon) with an energy lower than a potential barrier is able to transport through the barrier [1]. In general, the phenomenon varies between classical mechanics and quantum tunneling in terms of the ability to travel through the energy barrier. In classical mechanics, electrons should have sufficient energy, larger than the energy barrier, to reach the other side; otherwise, they are strictly blocked from transport [2]. However, when a quantum tunneling process is considered, electrons have the possibility to disappear from one side of the energy barrier and move to the other side without passing over the barrier. This process breaks the rules of classical mechanics and is derived from the wave-particle duality of matter in quantum mechanics $[3,4]$. The tunneling probability is considerably affected by the barrier thickness and would be extremely high at a 1-3-nm-thick barrier [5]. Unlike the classical confinement of carrier mobilities, the tunneling process is ultrafast but still remains inaccessible to the measurement for certain speed values. Quantum tunneling plays an essential role in advanced modern electronics and optoelectronics with various potential applications in tunneling field-effect transistors (FETs) [6-8], light-emitting devices (LEDs) [9-11], quantum computing [12-14], magnetization [15-17], and scanning tunneling microscopy (STM) [18-20]. These devices and equipments take advantage of quantum tunneling and demonstrate desirable performance with high-frequency operation, fast-speed capability, and low-power supply.

The semiconductor industry is fast approaching the fundamental limit of Moore's law [21]. To sustain rapid development, device engineering towards scaling down has intensely studied the use of high-k gate dielectrics [22-24], strained Si $[25,26]$, and short-gate geometries $[27,28]$. Among these device engineering techniques, quantum tunneling applications, with the ability to convert the characteristics, are promising substitutes for both speed and energy efficiency. Moreover, the device-asdesired concept has been proposed with considerable efforts to approach ideal device parameters. This concept falls under the domain of "Quantum Engineering," which

International Collaborative Laboratory of 2D Materials for Optoelectronics Science and Technology of Ministry of Education, Institute of Microscale Optoelectronics, Shenzhen University, Shenzhen 518060, China

* Correspondence authors (emails: hzhang@szu.edu.cn (Zhang H); maxyu1991@hotmail.com (Yu X)) 
has attracted an extraordinary amount of attention [29]. The prerequisite for accessing quantum tunneling is creating a tunneling barrier with the proper height. The creation of this barrier is a type of artificial work, which involves the tailoring of the band structure as desired [30-32]. In conventional $\mathrm{Si}, \mathrm{SiGe}$, or III-V techniques, 3D materials are used as the building blocks and are combined for band-structure engineering [33-35]. For a homojunction, it is not tenable to control a sharp doping profile at the sub-nanometer scale. A vague interface would induce a band edge tail, severely degrading quantum tunneling performance [36-38]. Simultaneously, heterojunction growth relies on molecular beam epitaxy (MBE) [39,40] or chemical vapor deposition (CVD) [41,42] techniques, which suffer from a limitation of material choices and the requirement to address lattice matching issues. To build multiple-quantum-well structures, the growth atmosphere is repetitively applied, which complicates the operation process to some extent $[43,44]$.

Beyond traditional techniques, two-dimensional (2D) van der Waals (vdW) layered materials are referred to as alternative candidates because of their atomically thin geometry and steep band edges [45-47]. Furthermore, because of a lack of dangling bonds on their surfaces, $2 \mathrm{D}$ materials can be easily stacked together, forming ultrathin homostructures or heterostructures without the requirement to consider lattice mismatch [48,49]. 2D materials are a large family with various electrical, optoelectrical, and magnetic properties [50-52]. Hexagonal (h)- $\mathrm{BN}$ is an insulator with a bandgap of $\sim 5.6 \mathrm{eV}$ [53], and is a superior $2 \mathrm{D}$ dielectric material that can be used as an intrinsic tunneling barrier [54]. Transition metal dichalcogenides (TMDs) are representative semiconductors in $2 \mathrm{D}$ materials that have various electron affinities and band gaps ranging from 0.4 to $2.0 \mathrm{eV}$ [55-57]. This allows the development of diverse heterostructures with type I (straddling gap), type II (staggered gap), or type III (broken gap) band alignments. Furthermore, recently emerging $2 \mathrm{D}$ materials, such as black phosphorus (BP) [58,59], graphdiyne [60,61], tellurene $[62,63]$, and MXene $[64,65]$, offer additional possibilities for quantum tunneling engineering. Furthermore, in integration research, wafer-scale production of $2 \mathrm{D}$ materials is now coming of age [66-69]. Because of their exceptional nature, $2 \mathrm{D}$ materials and their vdW heterostructures have considerable potential to present quantum tunneling. With a narrow vdW gap and abrupt band edges, vdW structure-based tunneling devices are believed to provide high tunneling performance, out- performing 3D devices because of their scaled-down thickness and shorter tunneling distance. The examination of $2 \mathrm{D}$ tunneling properties and devices is at its primary stage; additional studies will take this field another step forward.

In this review, we focus on quantum tunneling across $2 \mathrm{D} v \mathrm{vdW}$ heterostructures. We start with a discussion of tunneling transport by different mechanisms, then introduce various properties of $2 \mathrm{D} \mathrm{vdW}$ heterostructures, and finally extend the discussion to their appealing applications. The tunneling process can be classified into two types of electrical field-driven tunneling: direct tunneling (Fowler-Nordheim (FN) tunneling, band-to-band tunneling, quantum well, and Zener breakdown tunneling) and external-assisted tunneling of trap-assisted tunneling or tunnel magnetoresistance (TMR). In terms of creating a tunneling barrier for electron transport, structural engineering of tunneling devices has been developed towards band-to-band tunneling using tunable type II band alignment or intrinsic type III broken-gap alignment and the direct insertion of a large band-gap hBN barrier or magnetic insulator. By applying these features to device applications, various tunneling devices, such as tunnel diodes, tunnel FETs, superlattices, photodetectors, LEDs, memory cells, and spin valves, have been reported (Fig. 1).

\section{TUNNELING TRANSPORT IN 2D VDW HETEROSTRUCTURES}

In general, electron tunneling transport in $2 \mathrm{D}$ vdW heterostructures is similar to that in 3D devices. Depending on different materials, structures, thicknesses, and band alignments, electrons follow different tunneling mechanisms, which can be classified into either electrical field-driven tunneling or external-assisted tunneling. When a bias voltage is applied on the junction, the band alignment is tailored by the electrical field, inducing electron tunneling by direct tunneling, FN tunneling, band-to-band tunneling, superlattice and resonant tunneling based on the quantum well, or Zener breakdown tunneling. External-assisted tunneling includes trap-assisted tunneling and magnetic field-controlled spin tunneling, which are determined by varying the external environment. In addition to similar tunneling behavior, their atomically thin thickness endows $2 \mathrm{D}$ materials with desirable tunneling properties. Unlike band alignments in 3D junctions, 2D band alignments lack a depletion region and process the tunneling with a narrow tunneling barrier. In this section, the mechanisms underlying each type of tunneling process, which are the basic principles for 


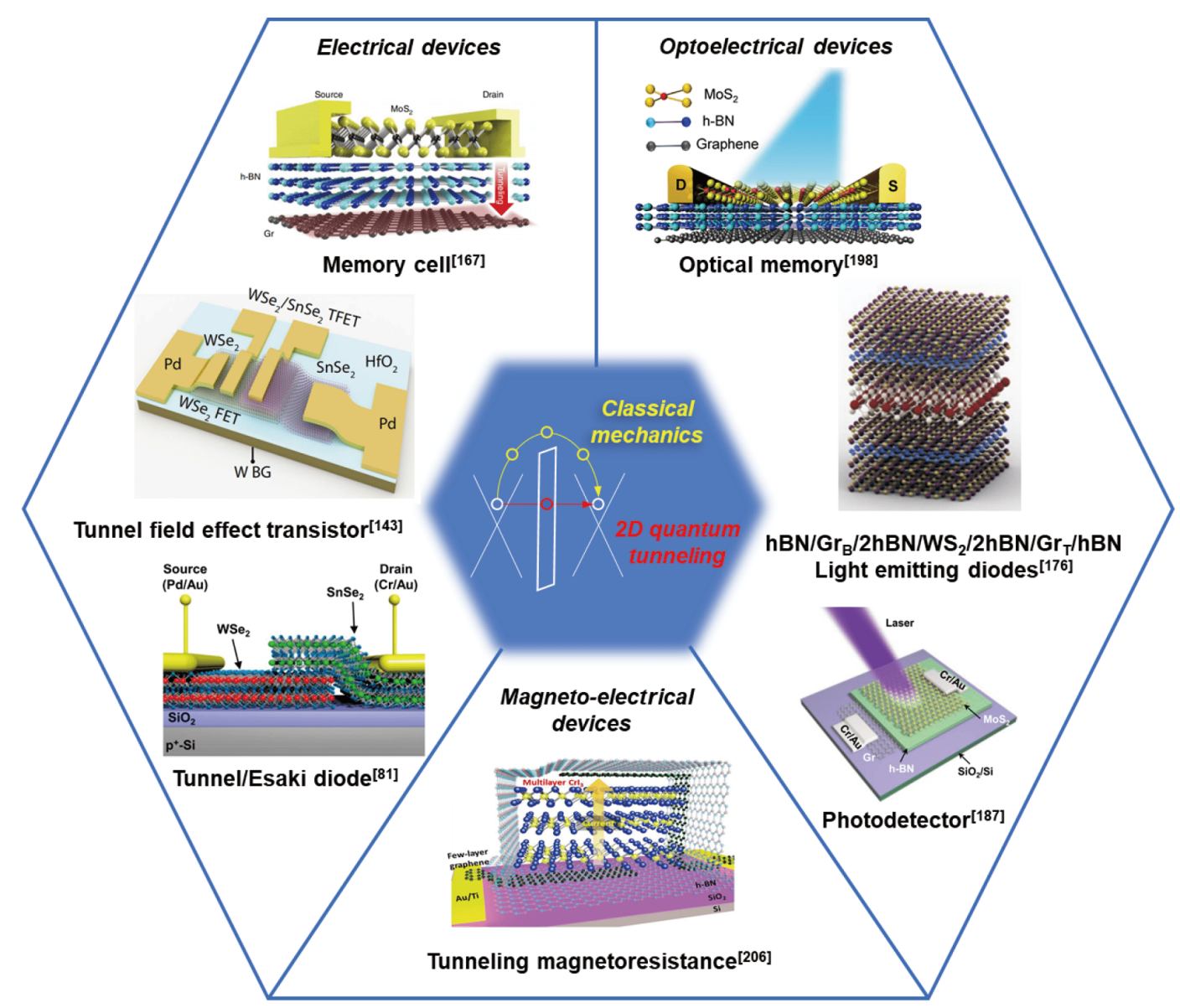

Figure 1 An overview of various 2D quantum tunneling applications in the research fields of electrical, optoelectrical, and magneto-electrical devices. Adapted with permission from Ref. [167], Copyright 2016, Springer Nature; [198], Copyright 2018, Wiley; [143], Copyright 2020, Springer Nature; [176], Copyright 2015, Springer Nature; [81], Copyright 2019, American Chemical Society; [187], Copyright 2016, American Chemical Society; [206], Copyright 2018, American Chemical Society.

the design of functional devices, are introduced.

\section{Direct tunneling and FN tunneling}

An electron has the ability to tunnel through a potential energy barrier in an arbitrary shape. As shown in Fig. 2a, a thin layer of h-BN is inserted between a bilayer graphene system to construct a metal-insulator-metal (MIM) structure, where the electron is able to tunnel from one graphene layer to the other across the h-BN barrier. At thermal equilibrium, the two monolayers of graphene possess an identical band structure; thus, the h-BN forbidden gap preserves the original rectangle shape. In the presence of an electric field, the band structure of $\mathrm{h}-\mathrm{BN}$ is easily tailored because of the thin nature of $2 \mathrm{D}$ materials. With different energy band conditions, the as-generated tunneling current is dominated by a different mechanism. The relationship between the electric field and generated tunneling current can be estimated using the Simmons approximation [70].

\section{Direct tunneling}

Fig. $2 \mathrm{~b}$ shows a source-drain bias of $V_{\mathrm{ds}}$, which is applied to the vdW heterostructure. Under the electrical field, the h-BN band structure is tailored into a parallelogram shape. In the region of small $V_{\mathrm{ds}}$, the electron travels across the h-BN barrier by direct tunneling, where the tunneling barrier width is the thickness of h-BN and not changed by the electric field. The direct tunneling current, $I_{\mathrm{DT}}$, follows the approximation:

$I_{\mathrm{DT}}(V)=\frac{A \sqrt{m \varphi_{\mathrm{B}}} q^{2} V_{\mathrm{ds}}}{h^{2} d} \exp \left[\frac{-4 \pi \sqrt{m^{*} \varphi_{\mathrm{B}}} d}{h}\right]$,

where $A$ is the effective contact area between the graphene and h-BN, $m$ is the free electron mass, $m^{*}$ is the 

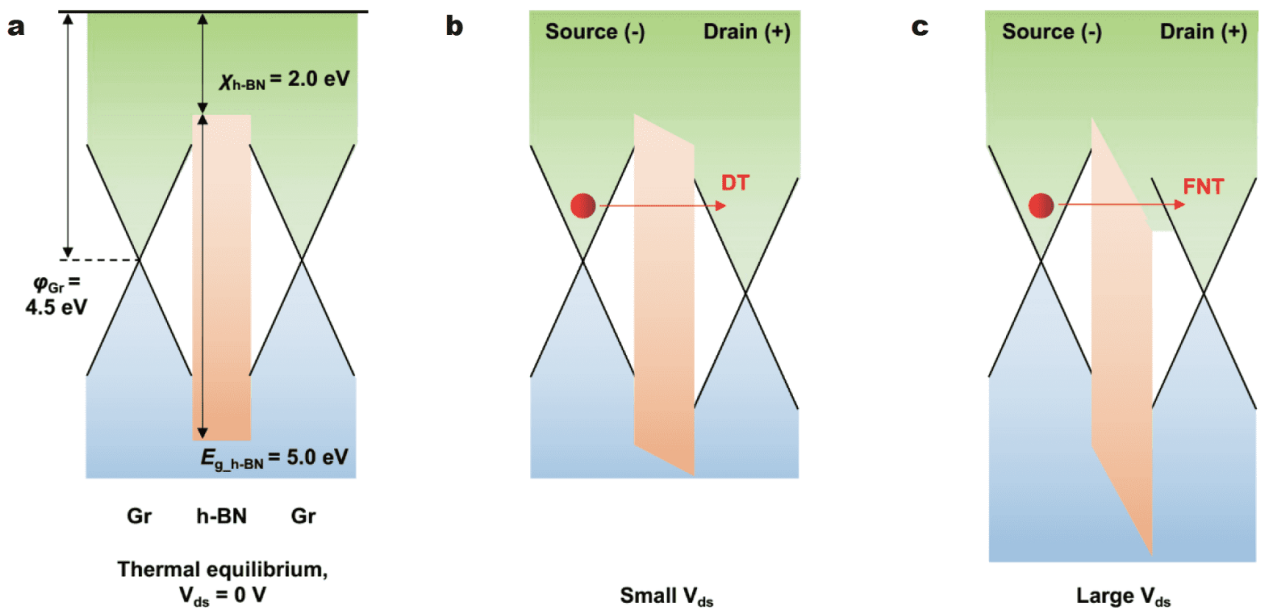

Figure 2 Energy band diagram of a graphene (Gr)/h-BN/Gr heterostructure (a) at thermal equilibrium, where $\chi$ is the electron affinity, $\varphi$ the is work function, and $E_{\mathrm{g}}$ is the band gap. (b) Direct tunneling at a small source-drain bias voltage $V_{\mathrm{ds}}$. (c) FN tunneling at large $V_{\mathrm{ds}}$. The red dot represents the electron for tunneling and the arrow indicates the tunneling direction.

effective electron mass, $\varphi_{\mathrm{B}}$ is the barrier height (from the Fermi level in graphene to h-BN), $d$ is the barrier width (the thickness of h-BN), $q$ is the unit charge of the electron, and $h$ is the Planck's constant.

\section{FN tunneling}

Driven by a larger bias, the $\mathrm{h}-\mathrm{BN}$ barrier is tailored, as shown in Fig. 2c. The h-BN forbidden gap retains the parallelogram shape; however, the triangle part is stretched to correspond with the graphene Fermi level, promoting electron tunneling transport. In this $V_{\mathrm{ds}}$ region, the barrier width is gradually reduced with the increase of bias voltage, increasing the tunneling probability. In such a case, the source-drain current is dominated by FN tunneling and $I_{\mathrm{FNT}}$ is expressed by the following equation:

$I_{\mathrm{FNT}}(V)=\frac{A q^{3} m V_{\mathrm{ds}}^{2}}{8 \pi h \varphi_{\mathrm{B}} d^{2} m^{*}} \exp \left[\frac{-8 \pi \sqrt{2 m^{*}} \varphi_{\mathrm{B}}^{3 / 2} d}{3 h q V_{\mathrm{ds}}}\right]$.

Because FN tunneling is induced by a high electric field, it causes a temperature increase in this region, resulting in thermionic emission dominating carrier transport [71]. Normally, it is difficult to directly distinguish between FN tunneling and thermionic emission without the help of temperature-dependent measurements. The derivation of Equation (2) is frequently used to investigate experimental current-voltage $(I-V)$ curves, providing a prominent clue for defining FN tunneling [72]. Equation (2) then becomes

$\ln \frac{I_{\mathrm{FNT}}(V)}{V_{\mathrm{ds}}{ }^{2}}=\ln \frac{A q^{3} m}{8 \pi h \varphi_{\mathrm{B}} d^{2} m^{*}}-\frac{8 \pi \sqrt{2 m^{*}} \varphi_{\mathrm{B}}^{3 / 2} d}{3 h q V_{\mathrm{ds}}}$.
From the basic relationship between FN tunneling dominated electron transport and current flow, the $\ln \left(I / V^{2}\right)$ versus $1 / V$ can be plotted with a negative slope near zero. However, when the plot lacks the negative slope, it suggests electrons obey the thermionic emission mechanism. The barrier height can then be estimated from the negative slope, $k$, in the linear regime [73,74], where

$k=-\frac{8 \pi \sqrt{2 m^{*}} \varphi_{\mathrm{B}}^{3 / 2} d}{3 h q}$.

\section{Band-to-band tunneling}

Band-to-band tunneling is electron tunneling between different bands in different materials, such as from the valence band $\left(E_{\mathrm{V}}\right)$ to the conduction band $\left(E_{\mathrm{C}}\right)$ in another region or material or when a reverse route is processed. It is the fundamental mechanism in tunnel diodes and tunnel FETs $[75,76]$. In 2D materials especially, their thin nature and quantum confinement effect strongly facilitate electron transport by tunneling.

\section{Tunnel diodes}

In conventional tunnel diodes, band-to-band tunneling occurs in a heavily doped $\mathrm{p}^{+}-\mathrm{n}^{+}$junction. Negative differential resistance (NDR) behavior can be clearly observed in the $I-V$ plot of a conventional tunnel diode, as shown in Fig. 3a, where the forward current is reduced upon the increase of bias voltage. With the NDR region, tunnel diodes exhibit considerable potential in applications as oscillators, high-frequency amplifiers, and in- 

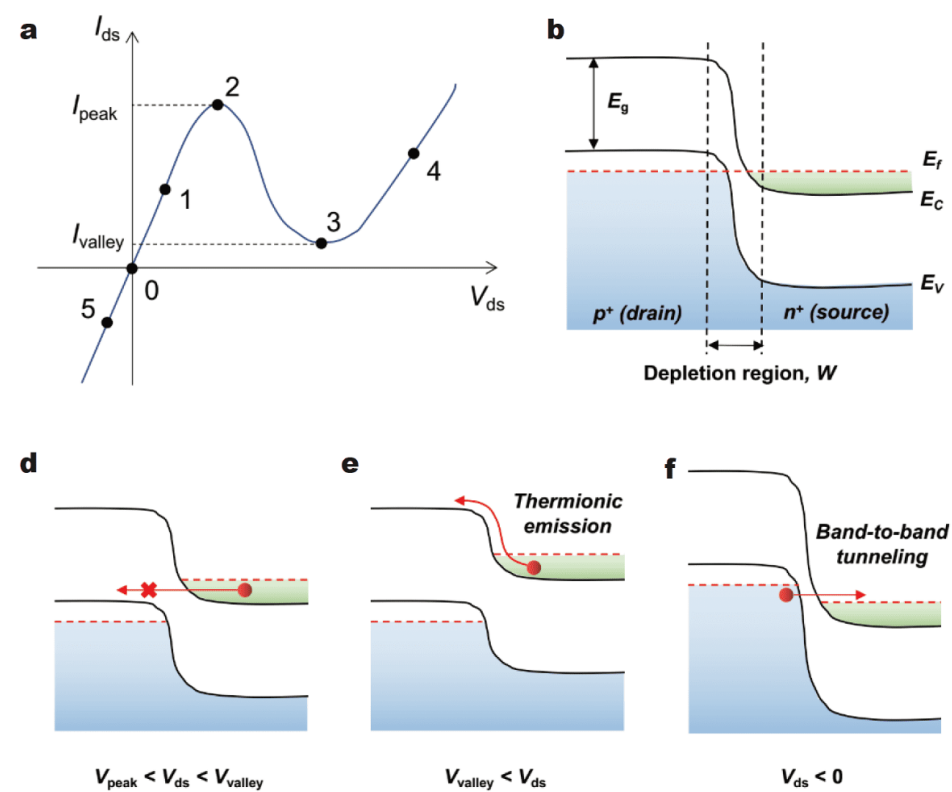

e

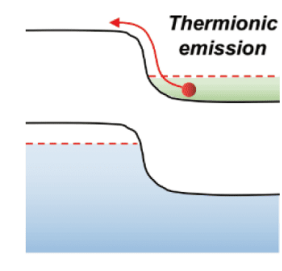

$V_{\text {valley }}<V_{\text {ds }}$

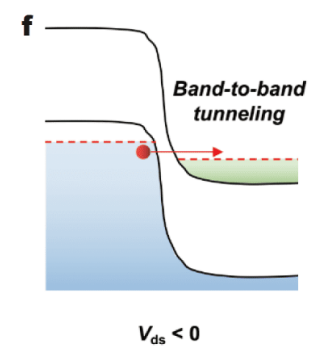

c

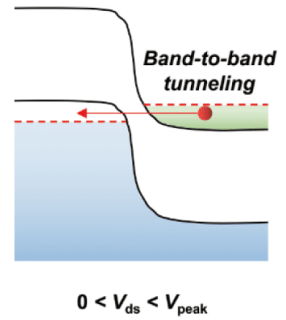

g

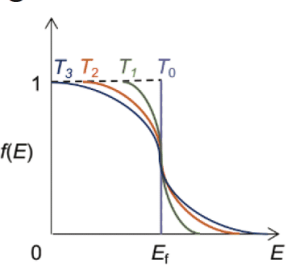

Figure 3 Tunnel diode based on band-to-band tunneling. (a) Diagram of $I-V$ characteristics with clear NDR behavior. (b) Band alignment of a $\mathrm{p}^{+}-\mathrm{n}^{+}$ junction at thermal equilibrium, where $W$ denotes the width of the depletion region, referred to as the width of the tunneling barrier. (c) Band

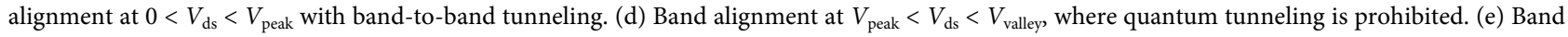
alignment at $V_{\text {valley }}<V_{\mathrm{ds}}$, where thermionic emission starts to dominate the carrier transport. (f) Band alignment at $V_{\mathrm{ds}}<0$ with reverse band-to-band tunneling current. (g) Diagram of the Fermi-Dirac distribution function $f(E)$; the temperature is set as $T_{3}>T_{2}>T_{1}>T_{0}$ and $T_{0}$ is the absolute zero.

tegrated trigger circuits [77-79]. Fig. 3b shows the energy band alignment at thermal equilibrium, where the Fermi level in the $\mathrm{p}^{+}$region enters the valence band while it is located in the conduction band of the $\mathrm{n}^{+}$region. Consequently, a depletion region appears between the two regions with a width,

$W=\left[\frac{2 \varepsilon_{\mathrm{s}} V_{\mathrm{bj}}}{q}\left(\frac{1}{N_{\mathrm{a}}}+\frac{1}{N_{\mathrm{d}}}\right)\right]^{1 / 2}$,

where $\varepsilon_{s}$ is the relative dielectric constant of the material, $V_{\mathrm{bi}}$ is the built-in electric field, $N_{\mathrm{a}}$ is the acceptor density in the p-region, and $N_{\mathrm{d}}$ is the donor density in the nregion. In terms of degenerate materials, $N_{\mathrm{a}}$ and $N_{\mathrm{d}}$ are large, which results in a narrow depletion region. The narrow depletion region increases the electron tunneling probability through the forbidden band to the other side, which can be evaluated using the Wentzel-KramersBrillouin (WKB) approximation [80]:

$T(E)=\exp \left[\frac{-4 \pi W \sqrt{m^{*} \varphi_{\mathrm{B}}}}{h}\right]$.

Equation (6) shows that the tunneling probability can be increased with reduced tunneling barrier width, height, and effective electron mass, which is an intrinsic characteristic of materials.
When the $\mathrm{p}-\mathrm{n}$ junction is driven by a small forward bias (Fig. 3c), the electron in the n-region conduction band is able to tunnel into the empty states in the p-region valence band, generating forward tunneling current from the p-region to the $\mathrm{n}$-region, indicated by Point 1 in Fig. 3a. When the Fermi level in the n-region aligns with the valence band edge in the p-region, the quantum states with equal energy on both sides reach a maximum. Consequently, the forward tunneling current meets its peak, indicated by Point 2 in Fig. 3a, namely, the peak current. When the bias voltage is increased in Fig. 3d, the conduction band in the n-region overlaps with the forbidden band in the p-region and electron tunneling is, therefore, largely prohibited, resulting in the decrease of forward tunneling current until reaching the valley, indicated by Point 3 in Fig. 3a. In this situation, the conduction band edge in the n-region is matched with the valence band edge in the p-region. The continuous increase of bias voltage can reduce the barrier height between the n-region and p-region. When the bias voltage is sufficiently large, the forward current would be dominated by thermionic emission transport, as shown in Fig. 3e and corresponding to Point 4 in Fig. 3a. Driven by a reverse bias in Fig. 3f, the energy bands in the p-region move up to where electrons can tunnel from the valence 
band into the other side, entering the conduction band in the n-region. The movement of electrons provides a tunneling current, as indicated by Point 5 in Fig. 3a. To evaluate a tunnel diode, there are two critical factors [81]. The first factor is the peak current density, which represents the amount of electron tunneling that decides the on-current in tunnel FET devices. The other factor is a large peak-to-valley current ratio, which is highly desired by oscillators for fast switching speed.

Carrier transport based on electron band-to-band tunneling or thermionic diffusion mechanisms is not easy to define in a simple $I$ - $V$ curve. One effective way is to fit the $I-V$ curves with corresponding equations. The current flow generated by quantum tunneling $\left(I_{\text {tunnel }}\right)$ or thermionic diffusion $\left(I_{\text {diffusion }}\right)$ can be theoretically calculated from the Equations (7 and 8) [82,83]:

$$
\begin{aligned}
I_{\text {tunnel }}= & \frac{2 \pi \alpha q}{h} \int_{E_{\mathrm{CN}}}^{E_{\mathrm{VP}}} \operatorname{DOS}_{p}(E) \times \operatorname{DOS}_{n}(E) \\
& \times\left[f_{p}(E)-f_{n}\left(E-q\left(V-I R_{\mathrm{s}}\right)\right)\right] \mathrm{d} E, \\
I_{\text {diffusion }}= & q I_{0}\left[\exp \left(\frac{V-I R_{\mathrm{s}}}{n k T}\right)-1\right],
\end{aligned}
$$

respectively, where $\alpha$ is a fitting parameter relating to the screen effect, $E_{\mathrm{VP}}$ is the valence band edge in the p-region, $E_{\mathrm{CN}}$ is the conduction band edge in the $\mathrm{n}$-region, $R_{\mathrm{s}}$ is a sum of all contact resistances (therefore $V-I R_{\mathrm{s}}$ stands for the voltage applied on the junction), $I_{0}$ is the reverse saturation current, $n$ is the ideality factor, $k$ is the Boltzmann constant, and $T$ is the temperature. $\operatorname{DOS}_{\mathrm{X}}(E)$ is the density of states (DOS) in each material, which is given by $\left(4 \pi m^{*}\right) / h^{2} . f_{\mathrm{X}}(E)$ shows the Fermi-Dirac distribution function of $\left(1+\exp \left[\left(E-E_{\mathrm{f}}\right) / k T\right]\right)^{-1}$, where $E_{\mathrm{f}}$ is the Fermi level.

One prominent difference between band-to-band tunneling and other carrier transport mechanisms is its temperature-dependent electrical properties [84]. The current contributed by thermionic emission, photon/ trap-assisted tunneling, or FN tunneling is dramatically reduced at low temperatures, whereas electron transport via band-to-band tunneling shows an opposite tendency with increased current. The temperature-dependent behavior can be probed by repeatedly measuring the NDR curves at different temperatures. In general, the tunnel diode fulfills two basic characteristics: (1) the device should be capable of maintaining the NDR behavior regardless of temperature variations, and (2) the peak current (tunneling current) density should increase with decreasing temperature. A diagram of the Fermi-Dirac distribution function, $f(E)$, is plotted in Fig. $3 g$ at different temperatures, where the temperature is set as $T_{3}>T_{2}>T_{1}$ $>T_{0}$, and $T_{0}$ is absolute zero. At room temperature, electrons can be emitted above the Fermi level by thermal motion, reducing the number of electrons for quantum tunneling. When the temperature decreases, the distribution of electrons becomes much closer to the Fermi level, increasing the probability of quantum states near the Fermi level to be occupied by electrons. Thus, the number of filled states below the Fermi level in the $n$ region increases while there are more empty states above the Fermi level in the p-region. Both the band-to-band tunneling current and the peak-to-valley current ratio increase. Another apparent feature in the $I-V$ measurements is the positive shift of the peak voltage at low temperatures, attributed to the increase in contact resistance at the metal-semiconductor junction [85]. NDR characteristics in vdW heterostructures, nevertheless, are primarily observed at low temperatures as room temperature behavior is frequently overwhelmed by thermal emission. In addition to the high performance, approaching room temperature stable NDR diodes is equally important and could develop their applications further.

\section{Tunneling FET}

The conventional FET, which uses the metal-oxidesemiconductor structure, is facing various challenges regarding scaling down and reducing energy consumption. The carrier injection and transport are restricted by thermionic emission, where the sub-threshold swing (SS) of an FET is limited to a minimum of $2.3 \mathrm{kT} / \mathrm{q}=$ $60 \mathrm{mV} \mathrm{dec}^{-1}$ at room temperature [86]. This indicates that, when an on/off current ratio of $10^{8}$ is required in the device, an operation voltage of $8 \times 60 \mathrm{mV}=0.48 \mathrm{~V}$ should be applied to fully switch the circuit at high speeds. Nevertheless, because of the physical limitations of scattering, trapping, or screening, an ideal SS of $60 \mathrm{mV} \mathrm{dec}{ }^{-1}$ is not possible in conventional FETs at room temperature as low temperature is normally required to reduce SS [87]. Thus, it is important to examine other mechanisms of carrier transport and injection to overcome the ideal value of $60 \mathrm{mV} \mathrm{dec}{ }^{-1}$. Considering this, band-to-band tunneling is adopted, which relies on extrafast quantum tunneling and breaks through the limitation of thermionic emission [88].

In conventional devices, $\mathrm{Si}, \mathrm{Ge}$, and III-V semiconductors have been adopted to fabricate tunnel FETs. Similar to tunnel diodes, a $\mathrm{p}^{+}-\mathrm{n}^{+}$junction is developed to process electron band-to-band tunneling. An intrinsic semiconductor is introduced between the $\mathrm{p}^{+}$region and 
$\mathrm{n}^{+}$region, forming a $\mathrm{p}^{+}-\mathrm{i}-\mathrm{n}^{+}$homojunction device structure. The electrical field effect is applied to the intrinsic part of the device to tune the Fermi level and thus tailor the band alignment in the homojunction. The $\mathrm{p}^{+}$and $\mathrm{n}^{+}$ regions are drain and source terminals, respectively. Fig. 4a shows the band alignment at thermal equilibrium, where the device is in the off state [89]. When the intrinsic part is gated by a positive voltage, the energy bands move downwards, bridging the tunneling channel between the $\mathrm{p}^{+}$region and $\mathrm{n}^{+}$region and the device is now switched into an on state. The tunneling probability for a tunnel FET is estimated from the basic equation [90]:

$T(E) \propto \exp \left[-\frac{8 \pi \sqrt{2 m^{*} E_{\mathrm{g}}^{3 / 2}}}{3 q h\left(E_{\mathrm{g}}+E_{\phi}\right)} \sqrt{\frac{\varepsilon_{\mathrm{i}}}{\varepsilon_{\mathrm{ox}}} t t_{\mathrm{ix}}}\right]$,

where $E_{\mathrm{g}}$ is the bandgap, $E_{\varnothing}$ is the energy range for tunneling, $\varepsilon_{\mathrm{i}}$ and $t_{\mathrm{i}}$ are the dielectric constant and thickness of the semiconductor, respectively, while $\varepsilon_{\mathrm{ox}}$ and $t_{\mathrm{ox}}$ are those for the gate oxides, respectively.

A perfect crystal of bulk material has a band-edge sharpness. Tunneling devices based on a traditional Si or Ge homojunction require ultra-high doping in the appointed region; however, this is not tenable for controlling a sharp doping profile at the sub-nanometer scale. Ultra-high doping would deteriorate the homojunction band edges, enlarging the screening tunneling length and tunneling barrier width. Moreover, 3D heterostructures suffer from fabrication complexity along with lattice matching concerns, which is not easily accessible for integrating desired materials. However, 2D materials are promising substitutes because of their abrupt band edges with an absence of surface dangling bonds. The combination of different 2D semiconductors allows various stacks with type III band alignment, as shown in Fig. 4b
[91-93], which is the prerequisite for band-to-band tunneling. Referring to their operation mechanisms, electron tunneling in $2 \mathrm{D}$ devices is generally similar to that in $3 \mathrm{D}$ devices, where the electron is able to tunnel from band to band when driven by an external bias. A difference from $3 \mathrm{D}$ homojunctions or heterojunctions is that due to the atomically thin nature of $2 \mathrm{D}$ materials, $2 \mathrm{D}$ heterojunctions lack a depletion region. Possessing steep band edges and narrow tunneling barrier widths (vdW gaps), the tunneling probability in 2D tunneling devices increases to a large extent, and they are expected to offer high performance, such as high tunneling current and peak-tovalley current ratio.

\section{Quantum well}

As shown in Fig. 5a, a quantum well is generated in semiconductor systems by inserting a thin layer of material with a narrow bandgap between two layers of materials with a wider bandgap $[94,95]$. One crucial step in forming a quantum well is controlling the thickness of the thin layer. Generally, carriers in the semiconductors are free to move in three dimensions. When the thickness of the thin layer is comparable to the electron de Broglie wavelength, carriers are confined and forced to occupy a $2 \mathrm{D}$ planar region. Because of the sudden change in the energy level across the heterostructure, an electron in the conduction band tends to have lower energy within the quantum well (marked as Region II in Fig. 5a) than in regions I or III (surrounding Region II) and is therefore trapped in the well. Moreover, the holes are trapped in the potential well created in the valence band. Because the width of the quantum well is within the mean free path of carriers, it produces a potential well in the heterostructure along the $x$-direction, and the quantum confinement of a
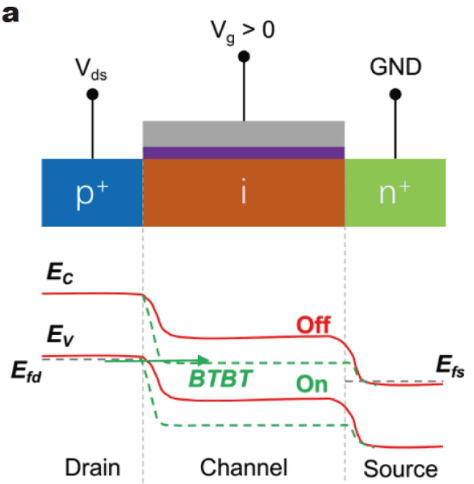

b

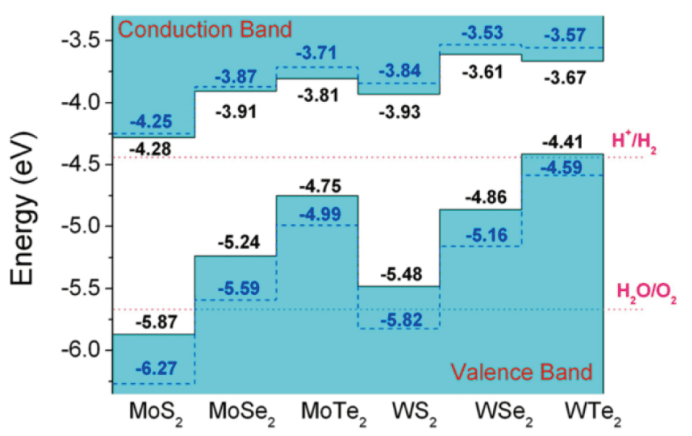

Figure 4 Tunnel FET based on band-to-band tunneling. (a) Schematic showing the tunnel FET structure of a $\mathrm{p}^{+}$-i-n ${ }^{+}$junction (top panel) and band alignment diagrams with on/off switch (bottom panel). (b) Band structure comparison of several 2D semiconductors. Adapted with permission from Ref. [92], Copyright 2013, American Institute of Physics (AIP) Publishing. 


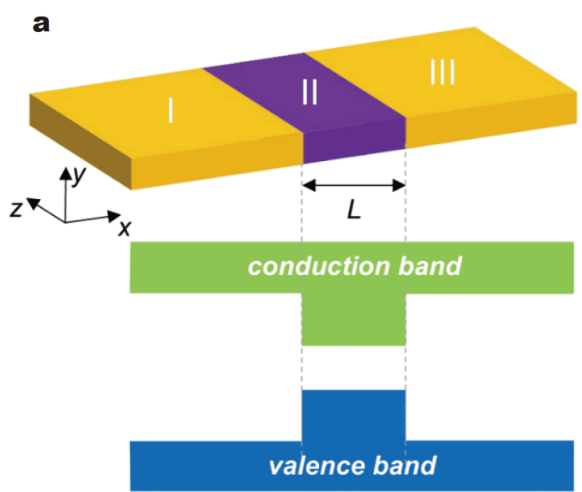

c

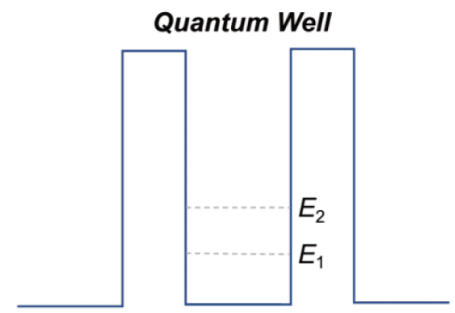

b
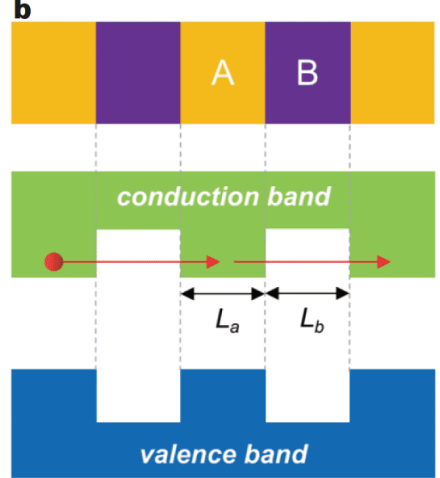

(ii)

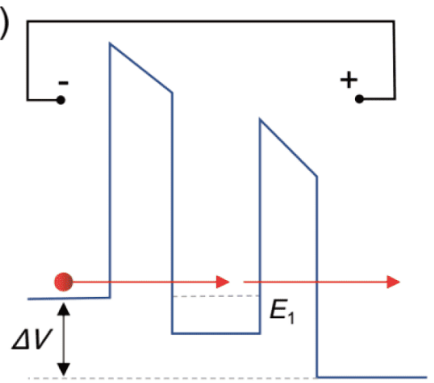

Figure 5 Quantum well. (a) Diagram of a quantum well, where materials I and III have wider band gaps and material II has a narrow band gap. The quantum well is created at the material II region. (b) Diagram of a superlattice, where materials A and B are grown in an alternating sequence. (c) Diagram of a RTD with band structure at (i) thermal equilibrium and (ii) external bias of $\Delta V$.

carriers occurs in the 2D $y$ - $z$ plane, which is parallel to the quantum well. The quantum confinement effect confines the carrier wave function in one dimension, and the energy states in the quantum well do not follow the previous parabolic curve and are instead discrete. In such a situation, the conduction band and valence band are split into sub-bands. With the quantum well mechanism, various devices and applications, such as quantum well lasers [96], photodetectors [43], superlattices, and resonant tunneling diodes (RTDs), have been successfully developed. Compared with conventional devices, quantum well devices possess a much faster speed and operate much more economically. Based on the fundamental properties of a quantum well, a 2D material has become a competitive candidate as a superior interlayer material because of its naturally atomic-scale thickness.

\section{Superlattices}

A superlattice is a periodic heterostructure composed of repeated quantum wells, where two types of semiconductors (denoted as A and B in Fig. 5b) with different bandgaps are alternately grown $[97,98]$. The energy band structure is, therefore, a period series of quantum wells. In the superlattice, the thickness of each single crystal layer $\left(L_{\mathrm{A}}\right.$ and $\left.L_{\mathrm{B}}\right)$ is controlled to be few or few tens of nanometers, where semiconductor $A$ with a narrow bandgap and semiconductor $B$ with a wider bandgap must be simultaneously sufficiently thin. Consequently, semiconductor A forms the quantum well that confines carriers, while semiconductor B serves as the tunneling barrier, allowing carriers to tunnel through the multiple quantum wells. An addition of $L_{\mathrm{A}}+L_{\mathrm{B}}$ is the periodic length in the superlattice, which is much longer than every single lattice constant. The thin barrier between the adjacent quantum wells promotes the wave functions in the neighboring wells to couple together; thus, the discrete energy states in each quantum well are extended, forming delocalized minibands. When a thick barrier is grown, the coupling between the adjacent wells is weak, whereas multiple quantum wells are generated rather than the superlattice. The electrons move along the material while alternating direction, which induces oscillating in the heterostructure that can be applied to fabricate diverse types of microwave devices [99] and high-power LEDs [100].

$R T D$

A RTD is a $2 \mathrm{D}$ diode fabricated from a resonant tun- 
neling structure in which electrons are able to escape from quantum confinement by tunneling through barriers via resonant energy states $[101,102]$. As shown in Fig. $5 c(i)$, one general structure is a double barrier containing a single quantum well surrounded by two thin layers of barriers. In the quantum well, the band edge is discontinuous and the energy states that carriers can occupy are discrete, as $E_{1}, E_{2}, \ldots$, etc., from the bottom up. This causes the prominent characteristics in RTDs with the presence of an NDR region in $I-V$ measurements. A small external bias, from right to left, is applied to the device in Fig. 5 c(ii). When the energy level in the left region aligns with the energy level $E_{1}$ in the quantum well, electrons can tunnel from left to right, forming a forward tunneling current. Because the electron tunneling probability reaches a peak value at the resonant energy level, the peak current thus appears when the conduction band in the left region matches the $E_{1}$ level. With the additional increase in the bias voltage, electrons supplied from the emitter region are blocked from resonant tunneling, leading to a reduction in tunneling current. Until the energy level in the left region meets the next energy level of $E_{2}$, another peak current would appear in the $I-V$ curve. Based on this mechanism in the resonant tunneling structure, multiple peaks can be observed in the $I$ - $V$ curve [103]. Unlike the band-to-band tunnel diode, the RTD has a nearly origin-symmetric $I-V$ curve. In real devices, RTD performance would be influenced by external factors, including materials, structures, the band edge, defects, and temperature, which induces a slight variation of peak points in both reverse and forward directions.

Hence, RTD is an open quantum system in which the energy states are scattered but continuously distributed in energy space, rather than having bound states with a discrete energy spectrum, as obtained in quantum wells.
These quasi-bound states used to increase electron tunneling across two thin barriers are the resonant states. The operation speed in devices can be evaluated by the electron tunneling time, which is related to the lifetime of quasi-bound states. These states are in a reverse proportional relationship with the width of the resonant energy level. It has been confirmed that the frequency in the RTD can reach up to the terahertz range, which indicates the tunneling time is shorter than a picosecond. This fast operation speed is extremely favorable for dealing with digital signals. Furthermore, the easy fabrication process and compatibility for high flexibility make the RTD a promising quantum device in modern physics and electronics.

\section{Zener breakdown tunneling}

In a $\mathrm{p}-\mathrm{n}$ diode, because of the balance of carriers' diffusion and drift, a depletion region appears at the $p-n$ junction. The depletion region is narrowed under a forward bias, while a reverse bias widens this region. When the applied reverse bias voltage is sufficiently large, a sufficiently strong electric field across the junction enables carriers to tunnel through the depletion region, as shown in Fig. 6a. The electrons tunnel from the valence band in the p-region to the conduction band in the nregion, generating multiple free minority carriers in a very short time period. Consequently, the reverse tunneling current is suddenly increased, as shown in Fig. 6b, which breaks down the device. In semiconductor electronics, this type of diode is called Zener diode, and the electrical breakdown mechanism is ascribed to the Zener effect $[104,105]$. Zener breakdown is a reversible breakdown process that disappears when the bias is removed. Based on these fundamental mechanisms, Zener diodes using $2 \mathrm{D}$ vdW junctions are believed to be superior to $3 \mathrm{D}$
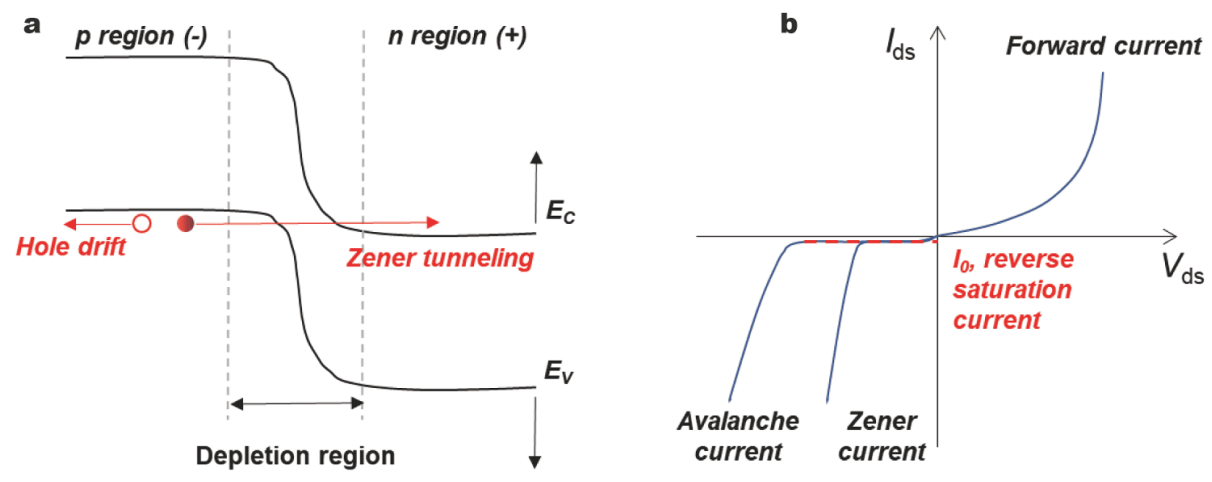

Figure 6 Zener tunneling breakdown effect. (a) Diagram of band structures in the case of Zener breakdown. (b) $I$ - $V$ comparison of Zener breakdown and Avalanche breakdown. 
junctions as the ultrathin depletion layer of the $2 \mathrm{D} v \mathrm{vdW}$ junction can efficiently increase Zener tunneling probability. This has been presented in photodiodes working in the Zener tunneling regime, which exhibit higher photoresponsivity than those of normal Si p-n diodes [106].

Another breakdown effect in $\mathrm{p}-\mathrm{n}$ diodes is the avalanche effect, whereby a sudden increase in the reverse current is observed [107]. There are certain differences that allow one to distinguish between the Zener and avalanche effects. During the avalanche breakdown process, minority carriers in the transition region are accelerated by the electrical field, providing sufficient energy for collision with bounded electrons and, therefore, numerous mobile electron-hole pairs are produced. Zener tunneling and avalanche breakdown could take place independently or simultaneously [108]. Because of the different breakdown mechanisms, a threshold voltage, $V_{\text {th }}$, where $V_{\text {th }}=4 E_{\mathrm{g}} / q$, exists between the two effects. Driven by a reverse bias smaller than $V_{\text {th }}$, device breakdown is primarily induced by Zener tunneling, whereas the avalanche effect dominates the breakdown process when the bias is larger than $V_{\mathrm{th}}$. At a bias close to $V_{\mathrm{th}}$, breakdown occurs by a combination of these two effects. $V_{\text {th }}$ is primarily determined by the width of the depletion region. In moderately or heavily doped junctions with a relatively narrow depletion region, Zener tunneling usually contributes to the reverse current. Avalanche breakdown can then be triggered in a lightly doped junction with a wider depletion region. Another difference in these breakdown effects is their temperature-dependent behaviors. A decrease in temperature would increase the Zener current because of the quantum tunneling process of carrier transport, whereas avalanche breakdown is lesser with a positive temperature coefficient. In terms of device performance, a Zener diode benefiting from electron tunneling is much more stable compared with its avalanche counterpart, which highly relies on random collisions between sub-particles.

\section{Trap-assisted tunneling}

In addition to direct tunneling and FN tunneling, which are one-step tunneling processes, defects in the tunneling barrier serve as intermediate sites, allowing electron tunneling with two or more steps. Trap or defect-assisted tunneling is a common phenomenon in non-volatile memory cells where a high electric field is required to access fast write and erase cycles. By repetitively applying high voltage stress, defects are induced in the dielectric layer, which results in tunneling current even at low bias voltage. This is called stress-induced leakage current (SILC), which is the primary reason for poor retention times in the memory cells [109-111].

Trap-assisted tunneling has been extensively studied using multiple approaches, including three fundamental models: inelastic phonon-assisted tunneling, single trapassisted tunneling, and multiple trap-assisted tunneling, which are applicable depending on the degradation degree of the dielectric layer. In a highly degraded dielectric, the stimulation of SILC is modeled by inelastic phononassisted tunneling [112]. An electron from the cathode is first captured by a trap site, then released to the lower energy level of the trap with the emission of one or more phonons and emitted to the anode, thus completing tunneling via the trap site. Fig. 7 a shows the complete process and is an inelastic process because of the emission of phonons with energy; thus, electron energy is not preserved. In the single trap-assisted tunneling model, only one trap participates in the tunneling process [113]. The tunneling current at each trap site is separately calculated by trap capture or emission rates, and the total tunneling current is modeled as an addition of the trap occupancy function of the individual trap site. The single trap-assisted tunneling assumption is appropriate for the modeling of slightly degraded dielectrics or thin gate dielectrics. In terms of thicker dielectrics, high defect den-
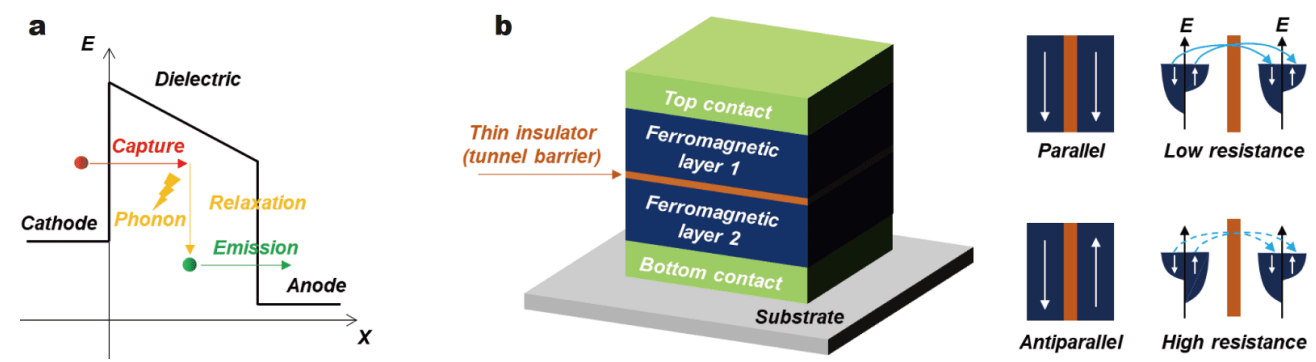

Figure 7 External-assisted tunneling. (a) Trap-assisted tunneling, including three steps of capture, relaxation with phonon, and emission. (b) Diagram of a magnetic tunnel junction showing magnetic field-controlled spin tunneling. 
sity results in the interaction of adjacent traps; therefore, the isolated calculation of each contributor is no longer sufficient $[114,115]$. In this situation, a multiple trapassisted tunneling model is used. During this process, two or more traps and the hopping of carriers between defect traps are considered in the modeling [116]. The oxide traps in the dielectric layer are filled by carriers, which induce a space charge with a shift of the threshold voltage in the transfer curve.

\section{TMR}

Both spin and charge properties of electrons can be utilized for the functionalization of devices; this is referred to as spintronics. A TMR device is a type of spintronic device composed of a magnetic tunnel junction (MTJ) [117-119]. The magnetoresistive effect can be observed via the change in resistance versus a varied magnetic field. Fig. $7 \mathrm{~b}$ shows a typical structure in which the TMR device comprises two ferromagnetic layers that are separated by a thin insulating layer. When the insulator is sufficiently thin, typically below a few nanometers, electrons are able to tunnel from one ferromagnetic layer to the other side. In this manner, the emergence of $2 \mathrm{D}$ materials with atomic thicknesses, such as $\mathrm{h}$-BN, is of great importance for scaled-down TMR devices with high quality and exceptional performance.

The working mechanism of TMR can be understood by Julliere's model, where two assumptions are simultaneously considered $[120,121]$. First, the spin properties of electrons are assumed to be preserved during the quantum tunneling process. An electron from one spin state in the top ferromagnetic layer can tunnel into the unfilled state with an identical spin in the bottom ferromagnetic layer. When two ferromagnetic layers are magnetized in parallel, electrons in the minority and majority spin states are able to tunnel into the corresponding minority and majority spin states on the other side, respectively. However, when two layers are antiparallel, electrons in the minority and majority spin states are accepted by the opposite states on the other side. Second, the tunneling process of up- and down-spin electrons is independent. Therefore, the resistance in each particular spin orientation is in isolation and proportional to the product of the DOS in the two ferromagnetic layers. The relative magnetization direction in the two ferromagnetic layers can be independently switched using an external magnetic field. When it is in parallel, the electron tunneling probability through the insulator is considerably higher than that in the two layers in antiparallel alignment. Consequently, a TMR device can be switched between two resistance states with a dramatic change in tunneling current. Low resistance $\left(R_{\mathrm{P}}\right)$ appears when the two magnetizations are in parallel, whereas an extremely high resistance $\left(R_{\mathrm{AP}}\right)$ is reported when the spin orientations are opposite each other. The spin-dependent tunneling determines the performance index in a TMR device via the MR ratio, which is given by $\left(R_{\mathrm{AP}}-R_{\mathrm{P}}\right) / R_{\mathrm{P}}$.

\section{QUANTUM TUNNELING TOWARDS 2D DEVICE APPLICATIONS}

In $2 \mathrm{D}$ tunneling devices, carrier transport relies on the quantum tunneling process, which is free of limitations induced by thermal excitation, discontinuous energy states, or high Schottky barrier height. Because of these characteristics, 2D devices have been developed for overcoming the SS restriction, efficiently injecting carriers, and suppressing the off current. In this section, we review various tunneling devices with different operating mechanisms. From the aspects of electronics, optoelectronics, and magnetoelectronics, tunneling devices based on broken band-aligned heterostructures, devices using h-BN barriers, and devices with magnetic insulators are introduced. Furthermore, quantum tunneling-assisted contact engineering is explained. 2D tunneling devices have introduced intriguing opportunities for realizing scaled-down, low-power, and fast-response devices such as diodes, FETs, LEDs, photodetectors, memory cells, and spin valves.

\section{Electrical devices}

\section{Esaki diodes}

The Esaki diode, which was discovered by Leo Esaki in 1957 , is a vital building block in very large-scale integration circuits (VLSI) [122]. Because of its quantum tunneling mechanism and the NDR behavior of its output curves, the Esaki diode is called a tunnel diode or NDR diode. To create band-to-band tunneling, components in $2 \mathrm{D} v \mathrm{dW}$ heterostructures are required to obey a specific band alignment of broken-gap type III or near brokengap type II. Fig. $4 \mathrm{~b}$ provides a practical band alignment simulation between various $2 \mathrm{D}$ semiconductors.

Fig. 8a shows an early studied Esaki diode based on a $2 \mathrm{D}$ vdW heterostructure using a bilayer $\mathrm{MoS}_{2} / \mathrm{WSe}_{2}$ system [83]. The $\mathrm{n}-\mathrm{MoS}_{2}$ and $\mathrm{p}-\mathrm{WSe}_{2}$ are artificially stacked together, forming the original type II band alignment shown in Fig. 8b. To independently modulate the carrier concentrations and Fermi level positions, additional dual gates are introduced into the device structure. By gating the $\mathrm{MoS}_{2}$ and $\mathrm{WSe}_{2}$ into the extremely n-type and p-type 

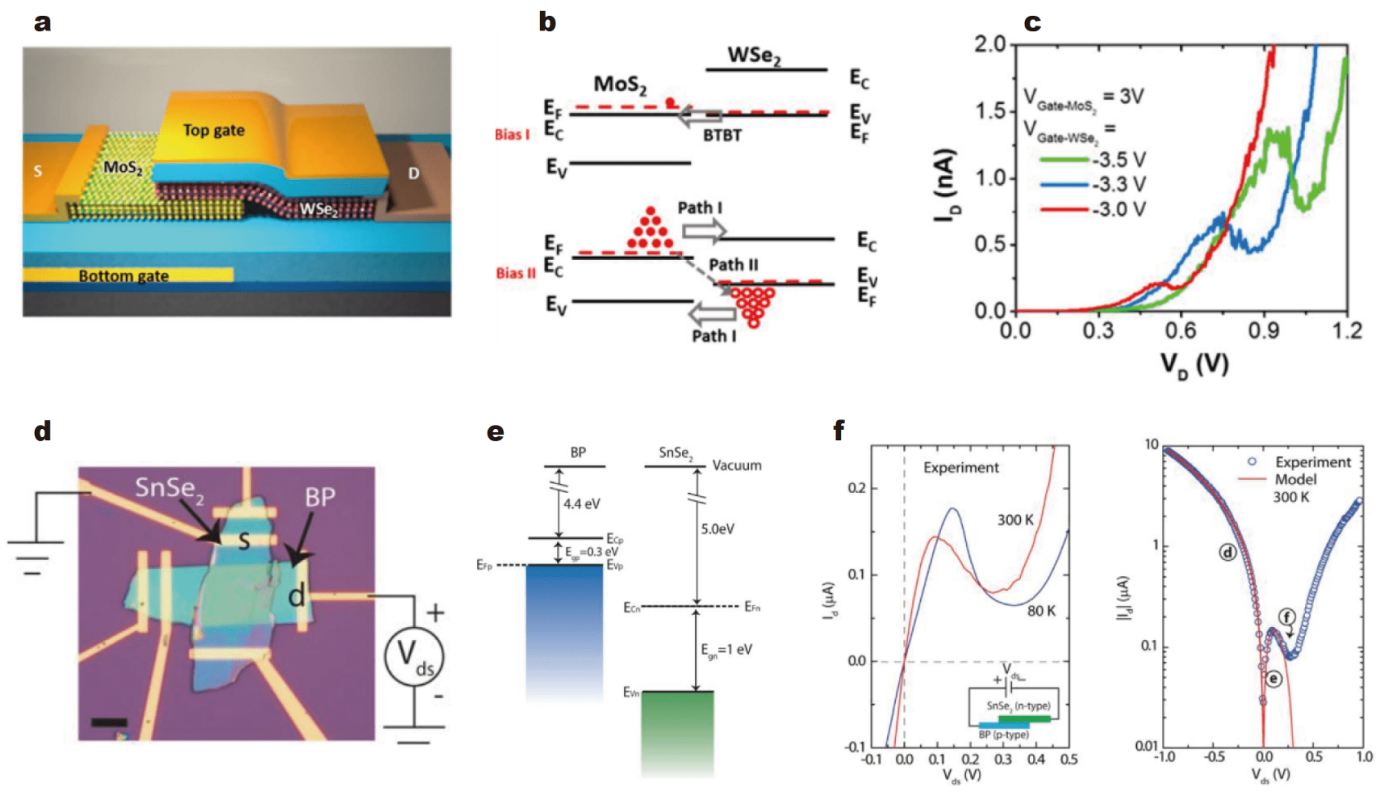

Figure 8 Esaki diodes. Type II-aligned $\mathrm{MoS}_{2} / \mathrm{WSe}_{2}$ heterostructure device (a) schematic possessing dual gates, (b) band alignments in the NDR regime, and (c) NDR behaviors at low temperature. (a-c) Adapted with permission from Ref. [83], Copyright 2015, American Chemical Society. Type III-aligned BP/SnSe $e_{2}$ heterostructure device (d) optical microscopy image, (e) band alignment at thermal equilibrium, and (f) stable NDR behavior at room temperature. (d-f) Adapted with permission from Ref. [124], Copyright 2015, American Chemical Society.

regions, respectively, an obvious NDR behavior is observed (Fig. 8c), indicating the realization of electron interlayer tunneling in the vertical TMD heterostructure. However, NDR behavior at room temperature is not tenable and only appears when the device is operated at low temperatures, which is attributed to violent thermal emission from the metal contact. Using the same structure, systematic analysis has been conducted to investigate interlayer tunneling in $\mathrm{vdW}$ heterostructures, including both vertical out-of-plane and horizontal inplane directions [123]. Energy band diagram simulations confirm that the effective bandgap at the edge of the overlapped region is much smaller than that in the overlapped region. This indicates that interlayer tunneling preferentially occurs in the planar direction rather than across the out-of-plane one. This is because of the nanoscale thinness of $2 \mathrm{D}$ materials, which cannot afford band bending along their thickness in the vertical direction and where the depletion region is primarily generated in the planar direction for a few micrometers. However, the dual gates used for electric field gating make the device fabrication process complex. Thus, there is an urgent ongoing search for an approach that not only removes the dual gates but also allows the formation of broken gap alignment. Based on fundamental research, intrinsic type III band alignment with broken-gap energy band offset has attracted wide attention as an additional process, such as electrostatic doping or chemical doping, and can be efficiently waived. Fig. $8 d$ shows a representative system using the $\mathrm{BP} / \mathrm{SnSe}_{2}$ heterostructure, where the $\mathrm{BP}$ valence band edge is $0.3 \mathrm{eV}$ higher than the $\mathrm{SnSe}_{2}$ conduction band in Fig. 8e [124]. This system realizes stable NDR behavior at room temperature with a peak-to-valley current ratio of 1.8 , which increases to 2.8 when the temperature decreases to $80 \mathrm{~K}$, as shown in Fig. 8f. Another representative system is that constructed by the $\mathrm{BP} / \mathrm{ReS}_{2}$ heterostructure [125]. High peak-to-valley current ratios of 4.6 and 6.9 are observed at room temperature and $180 \mathrm{~K}$, respectively. Moreover, by combining several type III vdW heterostructures in series, such as $\mathrm{BP} /\left(\mathrm{ReS}_{2}+\mathrm{HfS}_{2}\right)$ heterostructures, multiple peaks of NDR phenomena are available in a single device [126].

Vertical stacked 2D vdW heterostructures with homogeneity and band steepness at the interfaces are believed to offer exceptional performance in band-to-band tunneling. Among these heterostructures, the $\mathrm{WSe}_{2} / \mathrm{SnSe}_{2}$ system is predicted to yield a high tunneling probability because of the potential to form broken band alignment, which can reduce the voltage drop at the vdW gap [127]. Few devices have been fabricated [128,129]; nevertheless, none of the fabricated devices show stable NDR behavior at room temperature. Most 2D Esaki diodes demonstrate 
poor tunneling properties, inferior to those based on 3D materials [130,131]. Transmission electron microscopy (TEM) observations demonstrate clear evidence of an amorphous layer at the interfaces in Fig. 9a (marked by the green rectangle) [132], as well as in the $\mathrm{BP} / \mathrm{SnSe}_{2}$ system in Fig. 9b [124]. WSe ${ }_{2}$ is robust and resists degradation in ambient air, whereas BP is etched layer by layer by air oxidation with a distinct reduction in thickness [133]. The oxidation layer at the interfaces is thus ascribed to $\mathrm{SnSe}_{2}$ oxidation. The oxidation layer acts as a tunneling barrier, providing numerous traps, recombination sites, and scattering centers that severely degrade device performance. Benefitting from well-developed dry transfer approaches within a glovebox, this oxidation layer can be removed, resulting in a clean and ultrasharp interface [81]. Fig. 9c shows a TEM comparison of devices made in a glove box and the air. The device fabricated in an inert gas environment shows an extremely high tunneling current density of up to $1460 \mathrm{~mA} \mathrm{~mm}^{-2}$ (Fig. 9d). This is three to four orders of magnitude higher than previously reported values, and the peak-to-valley current ratio is over four at room temperature. The device at ambient conditions is unstable because of the random oxidation process at the interface. Hence, high heterointerface quality is important for achieving high device performance.

Unlike 3D tunnel diodes, which possess a depletion region that allows doping engineering to create a $\mathrm{p}^{+}-\mathrm{n}^{+}$ homo- or heterojunction [134], the depletion region along the vertical direction is absent in $2 \mathrm{D}$ heterostructures. Fig. 10 shows that when band bending at the contact junction is considered, the mechanism is slightly different than as introduced in the Section of LEDs. The Schottky barrier at the $\mathrm{Pd} / \mathrm{WSe}_{2}$ contact is considerably higher than the previously used $\mathrm{Cr} / \mathrm{WSe}_{2}$ contact, which largely suppresses thermionic emission current at the valley point. This enables the observation of NDR behavior at room temperature. Furthermore, encapsulating devices with an h-BN sandwiched structure could improve their performance. Note that additional improvements can be attributed to flat substrates and the prevention of impurities in the air [87]. This type of device possesses high quality with excellent potential for large tunneling properties, paving the way to realize tunnel FETs using an h-BN dielectric.

Another important engineering method for vdW heterostructures is the modulation of quantum tunneling to realize multifunctional applications. The underlying mechanism is attributed to the different carrier transport properties, which can be tailored by varying carrier a

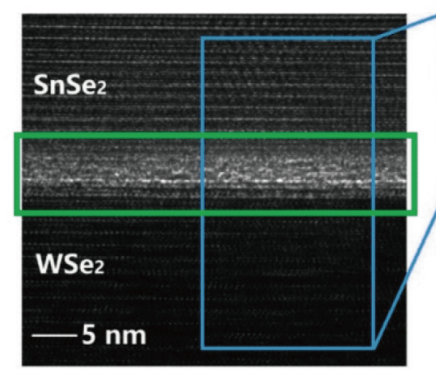

C

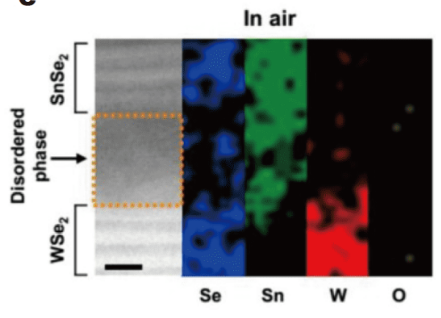

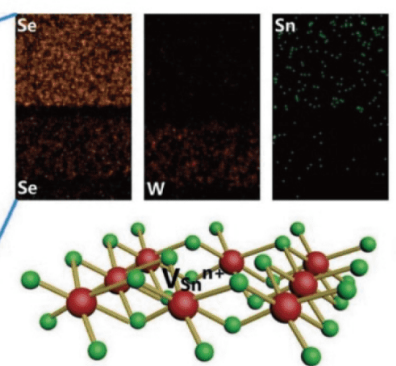

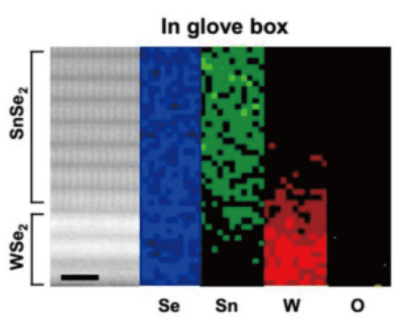

b

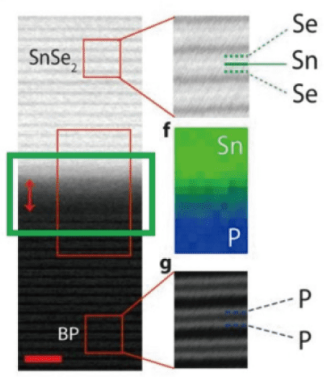

d

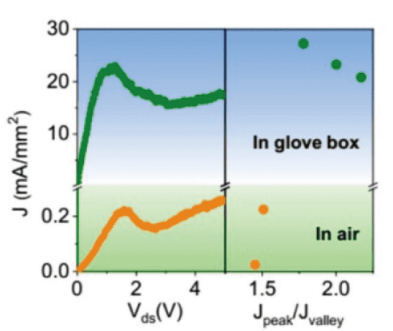

Figure 9 TEM cross-sectional images of a (a) $\mathrm{SnSe}_{2} / \mathrm{WSe}_{2}$ heterostructure. Adapted with permission from Ref. [132], Copyright 2017, Wiley; (b) $\mathrm{SnSe}_{2} / \mathrm{BP}$ heterostructure. Adapted with permission from Ref. [124], Copyright 2015, American Chemical Society; and (c) SnSe $/$ WSe ${ }_{2}$ heterostructure fabricated in air and in a glove box. (d) NDR performance comparison of devices made in air and in a glove box. (c, d) Adapted with permission from Ref. [81], Copyright 2019, American Chemical Society. 

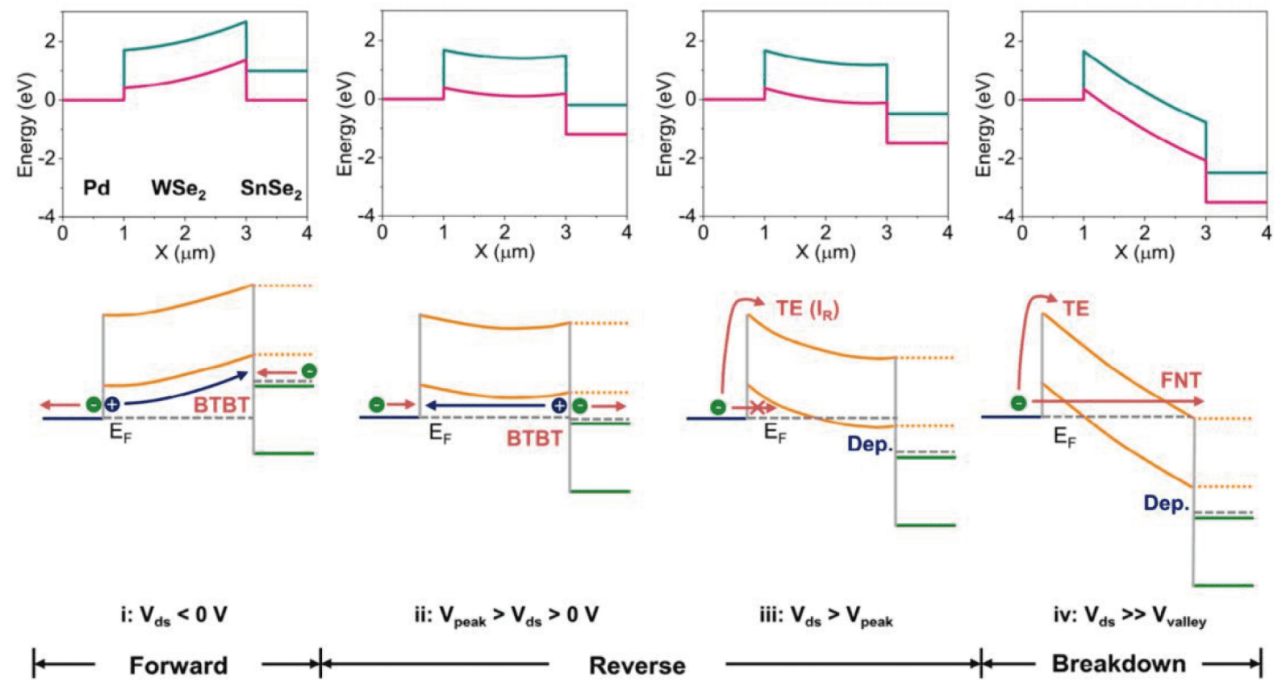

Figure 10 Band alignment simulations using Poisson's equation for the structure of $\mathrm{Pd} / \mathrm{WSe}_{2} / \mathrm{SnSe}_{2}$, under different source-drain bias voltages, which show the electron tunneling process in 2D vdW heterostructures. Adapted with permission from Ref. [81], Copyright 2019, American Chemical Society.

concentrations in the materials. This approach includes various methods such as electrostatic doping [83], chemical doping with an acceptor or donor [135], and phase transitions [136]. Furthermore, 2D materials have thickness-dependent band structures, and BP is one material with sensitive characteristics of the Fermi level, bandgap, and electrical properties [137]. As per the different thicknesses of BP, Fig. 11a, d show multiple functions are available in a $\mathrm{BP} / \mathrm{MoS}_{2}$ heterostructure, including the forward rectifying diode, backward rectifying diode, and Zener diode. Similar behavior can be reported in vdW heterostructures of $\mathrm{MoS}_{2} / \mathrm{MoTe}_{2}$ [138], BP/InSe [139], and $\mathrm{BP} / \mathrm{ReS}_{2}$, where additional linear conduction is observed when the BP thickness is $>100 \mathrm{~nm}$ [140]. To extend multifunctional devices into large-scale applications, CVD samples have been included in device fabrication [141]. The carrier concentration in monolayer $\mathrm{WSe}_{2}$ is precisely adjusted during the CVD synthesis process. During the CVD process, the vanadium dopant of an appointed concentration is mixed in the precursor, which provides a p-type doping effect with increased hole carrier concentration. By vertically stacking a layer of $\mathrm{SnSe}_{2}$ on $\mathrm{V}$-WSe $\mathrm{W}_{2}$, different band alignments are realized on the doping concentration, performing multifunctional diode behavior with tailored quantum tunneling (Fig. 11e).

\section{Tunnel FETs}

The continuous scaling down of Si-based transistors has largely boosted the semiconductor industry, which is important to the development of successful information techniques. However, because Moore's law meets its limitation, the semiconductor industry faces challenges for scaling down further. The first concern is the short channel effect. 2D semiconductors are promising candidates to replace Si because they are able to sustain device properties with a reduced channel length. Second, the power supply in conventional semiconductors is no longer reduced along with device scaling, which is attributed to the thermionic limitation in the SS. Band-toband tunneling transport can break the Boltzmann limit of the minimum SS of $60 \mathrm{mV} \mathrm{dec}^{-1}$ at $300 \mathrm{~K}$, enabling steep turn-on characteristics. Hence, the realization of tunnel FETs based on 2D materials is an essential step in reaching the scaling target without the exchange of a power penalty.

In general, 2D tunnel FETs can be developed from tunnel diodes, which possess band-to-band tunneling properties with proper band alignment. The on/off switch relies on the electrical field-affected transition in the band alignment where a degenerate material is always employed. A tunnel FET based on the $\mathrm{BP} / \mathrm{MoS}_{2}$ heterostructure integrated with ion gel dielectric has been fabricated and characterized [142]. When the Fermi level in the $\mathrm{MoS}_{2}$ channel is electrostatically modulated to close to the conduction band, interlayer tunneling is triggered. A sharp SS of $65 \mathrm{mV} \mathrm{dec}^{-1}$ was observed at room temperature, which improved to $51 \mathrm{mV} \mathrm{dec}^{-1}$ at $160 \mathrm{~K}$. By replacing the dielectric with the high-k material at a thinner thickness, a minimum SS of $37 \mathrm{mV} \mathrm{dec}^{-1}$ at room temperature was demonstrated in a tunnel FET based on 
$\mathbf{a}$

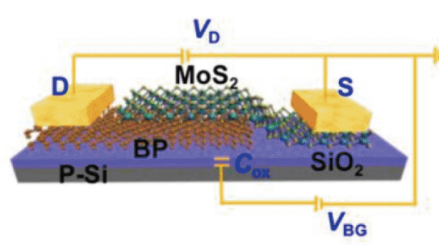

d

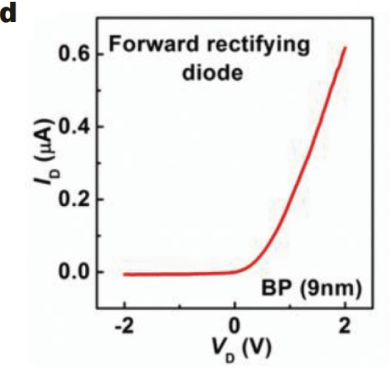

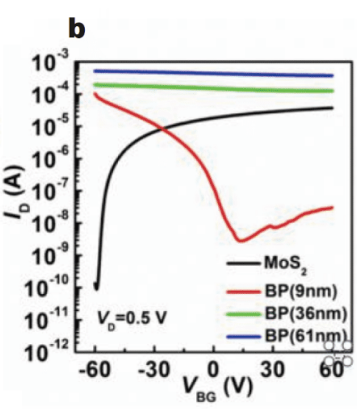

c
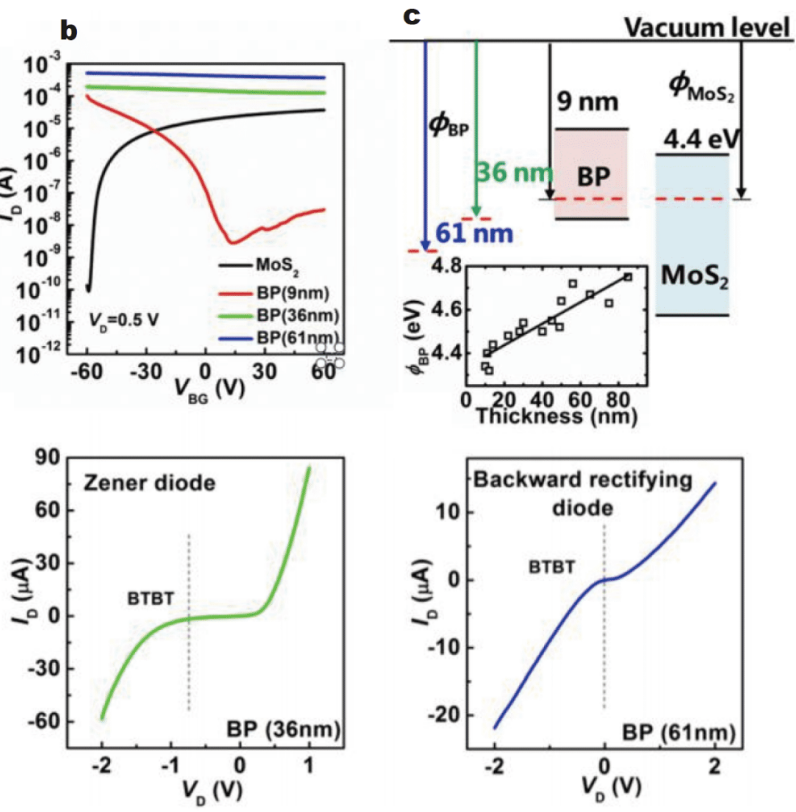

e
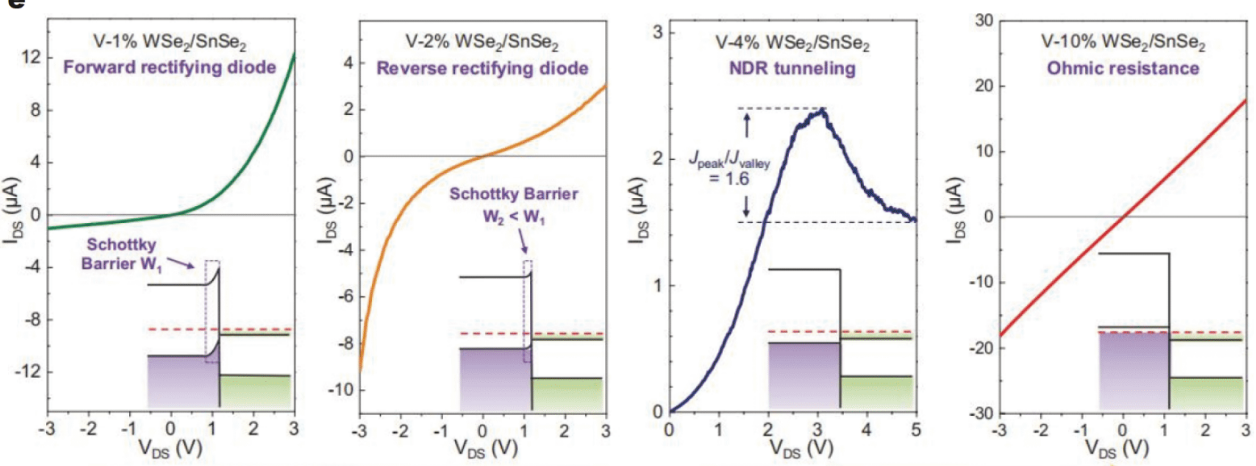

Increase $\mathrm{V}$-doping concentration in $\mathrm{V}$-doped $\mathrm{WSe}_{2}$ at $\mathrm{V}_{\mathrm{G}}=-90 \mathrm{~V}$

Figure 11 Multifunctional devices enabled by tailoring quantum tunneling. (a) Device structure diagram of a device based on the $\mathrm{BP} / \mathrm{MoS}_{2}$ heterostructure with (b) electrical characteristics of each component, (c) band alignments with $\mathrm{MoS}_{2}$ at different BP thicknesses, and (d) diverse diode behaviors, including a forward rectifying diode, Zener diode, and backward rectifying diode. (a-d) Adapted with permission from Ref. [137], Copyright 2017, American Chemical Society. (e) Device based on the vanadium doped $\mathrm{WSe}_{2} / \mathrm{SnSe}_{2}$ heterostructure showing diverse diode behaviors, including forward rectifying diode, reverse rectifying diode, NDR tunneling, and Ohmic resistance. Adapted with permission from Ref. [141], Copyright 2019, Wiley.

the $\mathrm{SnSe}_{2} / \mathrm{WSe}_{2}$ heterostructure [132,143]. High gate control switches the on/off current ratio to $>10^{6}$ with an on tunneling current larger than $10 \mu \mathrm{A}$.

Even a tunnel FET based on vertical vdW heterojunctions has resulted in a promising SS of sub- $60 \mathrm{mV} \mathrm{dec}^{-1}$; however, interface issues always limit device fabrication and result in experimental results far deviating from theoretical predictions [144]. Another type of 2D tunnel FET employs the traditional $\mathrm{p}-\mathrm{i}-\mathrm{n}$ structure, where a natural heterojunction is formed by spatially varying the thickness in one flake of BP $[145,146]$. Fig. 12a shows the device structure and band diagram, where a BP flake with two steps of thickness is adopted in the device. The electrical field is applied in the middle region of the flake to modulate the band alignment. Simultaneously, the bulk in the left region is not affected and the monolayer in the right region is connected to the drain. While sweeping the top or bottom gate, a tunneling channel is generated across the whole BP for electron transport, yielding an expected smaller SS. By avoiding interface problems, a record low SS of $22.9 \mathrm{mV} \mathrm{dec}^{-1}$ was achieved over four orders of current with a record on-current of 

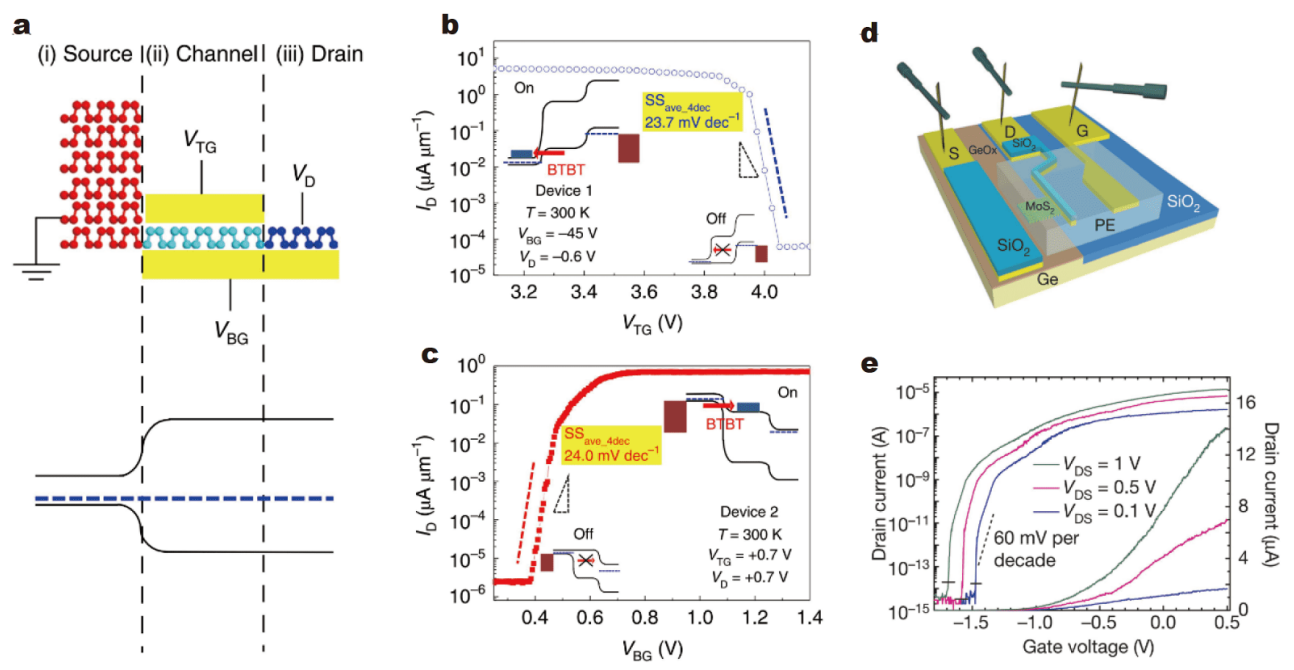

Figure 12 Tunnel FETs. (a) Device structure diagram and band alignment of a device based on the stepped BP homostructure with (b) p-type and (c) n-type transfer characteristics with steep SS. (a-c) Adapted with permission from Ref. [145], Copyright 2020, Springer Nature. (d) Device structure diagram of a device based on the $2 \mathrm{D} \mathrm{MoS} / 3 \mathrm{D}$ p-Ge heterostructure and (e) transfer characteristics with steep SS. (d, e) Adapted with permission from Ref. [149], Copyright 2015, Springer Nature.

$0.65 \mu \mathrm{A} \mu \mathrm{m}^{-1}$, as shown in Fig. 12b, c.

When $2 \mathrm{D}$ semiconductors serve as channel materials, they produce not only the expected gate control ability but also a smaller tunneling distance or tunneling barrier width, whereas 3D materials provide higher carrier concentration with higher mobility [147]. Because of these fundamental properties, it is promising to innovatively combine the maturity of $3 \mathrm{D}$ materials and the unique properties of 2D materials. This opens up unprecedented opportunities for designing advanced tunneling devices and increasing tunneling current without any concern for lattice matching issues. Based on this concept, $\mathrm{MoS}_{2}$ was selected as the $2 \mathrm{D}$ channel and degenerated $\mathrm{Ge}$ as the $3 \mathrm{D}$ source to be vertically stacked together, as shown in Fig. 12d [148,149]. This architecture enables efficient gate control to $\mathrm{MoS}_{2}$, a strain-free interface, a low tunneling barrier, and a large tunneling area. With near broken gap band alignment, the device shows steep turn-on properties with a minimum SS of $3.9 \mathrm{mV} \mathrm{dec}^{-1}$ and an average of $31.1 \mathrm{mV} \mathrm{dec}^{-1}$ for four orders of magnitude of current at room temperature while accessing a low power supply of $0.1 \mathrm{~V}$, as shown in Fig. 12e. This concept can be applied to exploit the Esaki diode using the $\mathrm{GaN} / \mathrm{MoS}_{2}$ heterojunction [150]. GaN is heavily doped n-type while $\mathrm{MoS}_{2}$ is doped by $\mathrm{Nb}$ atoms to be p-type. The peak current density in the device is up to $446 \mathrm{~A} \mathrm{~cm}^{-2}$ with a peak-to-valley current ratio of 1.2 at room temperature.

\section{RTDs}

Unlike the discrete energy states in 3D quantum wells, 2D semiconductors have negligible quantum capacitance and unique energy-momentum dispersion. By exploiting graphene/h-BN/graphene heterostructures, strong resonant peaks are observed in the output characteristics, as shown in Fig. 13a-c [151]. Thin h-BN serves as the tunneling barrier and two graphene layers act as the electrodes, creating the quantum well structure. The peak-to-valley current ratio was up to 4.0 at $7 \mathrm{~K}$ and the NDR behavior persisted at room temperature with a ratio of 1.3. The bias voltage at resonance can be affected by the relative crystalline orientation between these two graphene layers [152]. The resonance performance is highly related to interface issues, where an RTD possessing a directly synthesized heterostructure outperforms the manually stacked Gr/h-BN/Gr [153]. Resonant tunneling devices are tunable by an external magnetic field, wherein a stable oscillation can be produced in megahertz frequency. Furthermore, increased resonant tunneling is predicted by the theoretical calculation based on transistors with symmetric heterostructures of vertically stacked 2D semiconductors [154]. The unique resonant tunneling between two identical 2D materials enables the momentum conservation whereby stable NDR behaviors have been reported in several devices such as double bilayer graphene sandwiching the h-BN barrier $[155,156]$ or $\mathrm{WSe}_{2}$ layer [157].

When graphene is exchanged by TMD materials, an increased DOS with their resultant quantum capacitance enables gate tunable NDR in a very narrow resonance region. With the device structure of rationally controlled 
a

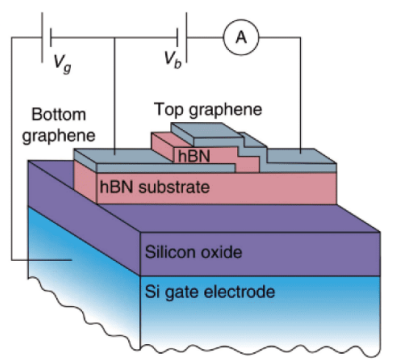

d

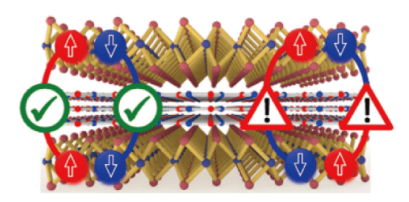

b
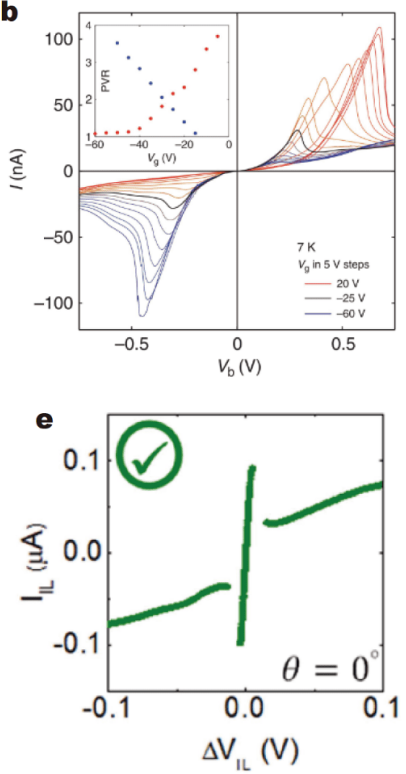
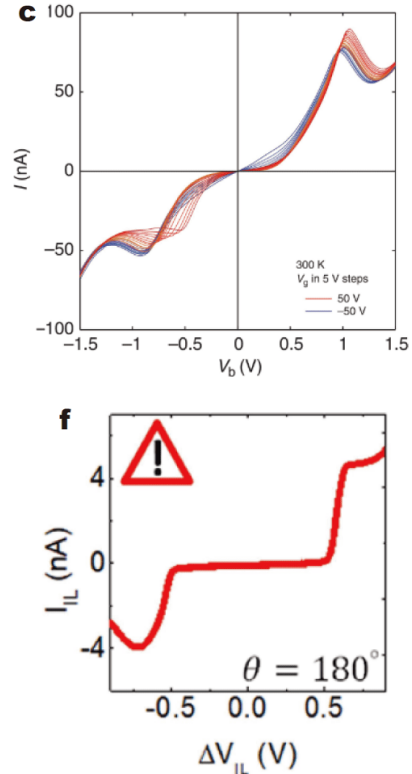

Figure 13 RTDs. (a) Device structure diagram of a device based on the graphene/h-BN/graphene heterostructure with output characteristics at (b) $7 \mathrm{~K}$ and (c) $300 \mathrm{~K}$, respectively. (a-c) Adapted with permission from Ref. [151], Copyright 2013, Springer Nature. (d) Device structure diagram of a device based on the rationally controlled $\mathrm{WSe}_{2} / \mathrm{h}-\mathrm{BN} / \mathrm{WSe} \mathrm{f}_{2}$ heterostructure with (e) resonance NDR behavior at a twist angle of $0^{\circ}$ and (f) no apparent primary resonance at $180^{\circ}$. (d-f) Adapted with permission from Ref. [158], Copyright 2018, American Chemical Society.

WSe $2 / \mathrm{h}-\mathrm{BN} / \mathrm{WSe}_{2}$ in Fig. 13d, the role of spin-valley conservation, in addition to the energy and momentum conservation during the interlayer tunneling, was experimentally probed [158]. When the twist angle between two monolayers of $\mathrm{WSe}_{2}$ is $0^{\circ}$, a sharp resonance appears near zero bias in Fig. 13e, attributed to momentum-spin conservation tunneling. Secondary resonances occur close to $\pm 0.5 \mathrm{~V}$, which is related to momentum-randomizing tunneling between spin-split valence bands. At a $180^{\circ}$ twist in Fig. 13f, there is no apparent primary resonance; however, an obvious secondary resonance appears because of the alignment of the lower and upper valence bands. Hence, NDR behavior can only be observed when two $\mathrm{WSe}_{2}$ are perfectly aligned, indicating that the resonant tunneling is dominated by spin and valley properties in $\mathrm{WSe}_{2}$. From another perspective, NDR behavior emerges when the bias voltage is closely equal to the $\mathrm{MoS}_{2}$ bandgap in the $\mathrm{MoS}_{2} / \mathrm{h}-\mathrm{BN} / \mathrm{MoS}_{2} /$ graphene heterostructure $[159,160]$. Resonant tunneling spectroscopy can thus be employed to characterize the bandgap in the material without the constraint of the common quasiparticle effect. Band splitting is observed in the forward tunneling current using a bilayer of $\mathrm{MoS}_{2}$, indicating the giant Stark effect induced by the vertical electrical field across vdW heterostructures.

\section{Memory applications}

Conventional floating gate memory cells based on 2D materials are operated by a control gate with three terminals [161-163]. Because of the requirements of fabricating gate electrodes, the carrier channels are relatively long, limiting high-density integration. Alternatively, a $2 \mathrm{D} v \mathrm{vW}$ heterostructure with graphene as the floating gate and an h-BN as the dielectric can be used. A nonvolatile programmable $\mathrm{p}-\mathrm{n}$ junction based on a $\mathrm{WSe}_{2} /$ $\mathrm{h}-\mathrm{BN} / \mathrm{Gr}$ heterostructure has been fabricated wherein the $\mathrm{WSe}_{2}$ channel is partially aligned with the floating gate $[164,165]$. This semi-floating gate exhibits unique features at different drain biases. Under a negative drain bias of $-1 \mathrm{~V}$, the carrier accumulation is processed in the floating gate, converting the $\mathrm{WSe}_{2}$ from a p-n junction to an $\mathrm{n}^{+}-\mathrm{n}$ junction. A positive bias of $1 \mathrm{~V}$ would block carrier storing, significantly affecting the on/off ratio of $10^{8}$ at negative bias and 10 at positive bias.

Another way of building two-terminal memory is proposed by assisting with an h-BN tunneling barrier [166]. In a practical structure, one layer of h-BN with proper thickness is placed between the floating gate of graphene and channel material. Both the charging and discharging processes in the graphene are conducted by direct tunneling of carriers through the h-BN barrier. 
Fig. 14a shows a memristor adopting the structure of graphen/h-BN/monolayer $\mathrm{MoS}_{2}$ with the operation mechanism in Fig. 14b [167]. By controlling the drain bias, the resistance of $\mathrm{MoS}_{2}$ is switched from high value (off state) to low value (on state) and then back to a high value (off state) for a dual sweep along the clockwise direction, thus attaining the current hysteresis loop shown in Fig. 14c. The device is thus operated with the several states shown in Fig. 14d: (i) programming, in which the electrons tunnel through the h-BN; (ii) reading, in which the electrons are stored in the graphene; (iii) erasing, in which the hole carriers enter into the graphene; and (iv) reading, in which the holes are confined inside the graphene. Here, the h-BN thickness is critical to promote high memory performance. It should be sufficiently thick to block leakage from the $\mathrm{MoS}_{2}$ in the reading process and thin enough for carriers to tunnel into the floating gate. Thickness-dependent measurements of h-BN indicate that $7.5 \mathrm{~nm}$ is a good choice in regard to the on/off ratio and retention time. Such a memory is free of a gate and dielectric, enabling reliable performance with high flexibility, stretchability, and scalability. The off current is extremely low, at $10^{-14} \mathrm{~A}$; thus, an ultrahigh on/off ratio up to $10^{9}$ is observed. The retention time is long, at $10^{4} \mathrm{~s}$, with stable endurance over $10^{5}$ cycles. Because of its reliable mechanical strength $[168,169]$, the excepted stretchability is preserved at $19 \%$ strain without apparent change in electrical properties, which is promising for applications in soft electronics. Furthermore, the exfoliated flakes can be exchanged with CVD-grown samples for large-scale commercialization in which h-BN is replaced by $\mathrm{Al}_{2} \mathrm{O}_{3}$ [170]. Therefore, exploring the tunneling properties in vdW heterostructures highly expands memory applications and assists the development towards neuromorphic computing [171].

\section{Optoelectrical devices}

\section{LEDs}

For practical applications, efficient light-emission is important for developing quantum optics. When semiconducting TMDs are thinned down to a monolayer, band structures evolve from the indirect bandgap in pristine bulk to a direct bandgap [172,173]. Benefiting from these properties, monolayer TMD materials are an ideal platform to examine the many-body complex of excitonic emission [174]. Because of the atomically thin nature of $2 \mathrm{D}$ materials, it is difficult to efficiently inject carriers with the conventional p-n junction structure used in 3D LEDs [175]. To overcome this limitation, a quantum well structure based on a vdW heterostructure was developed in which carriers can be injected via tunneling
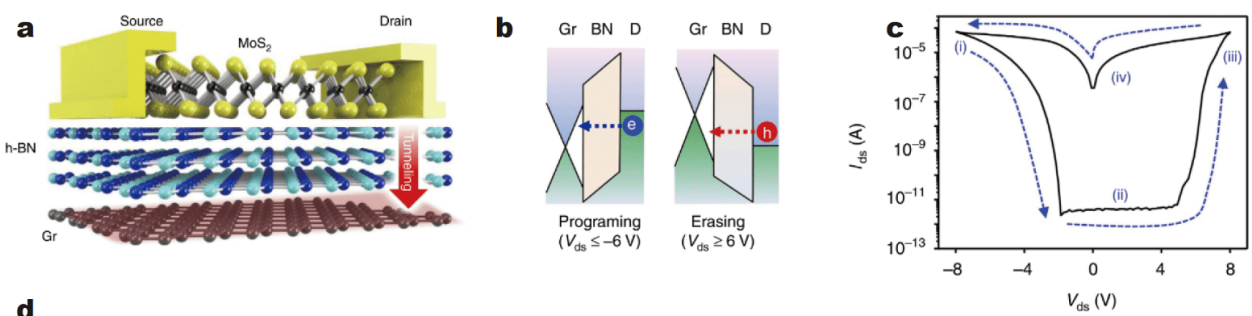

d
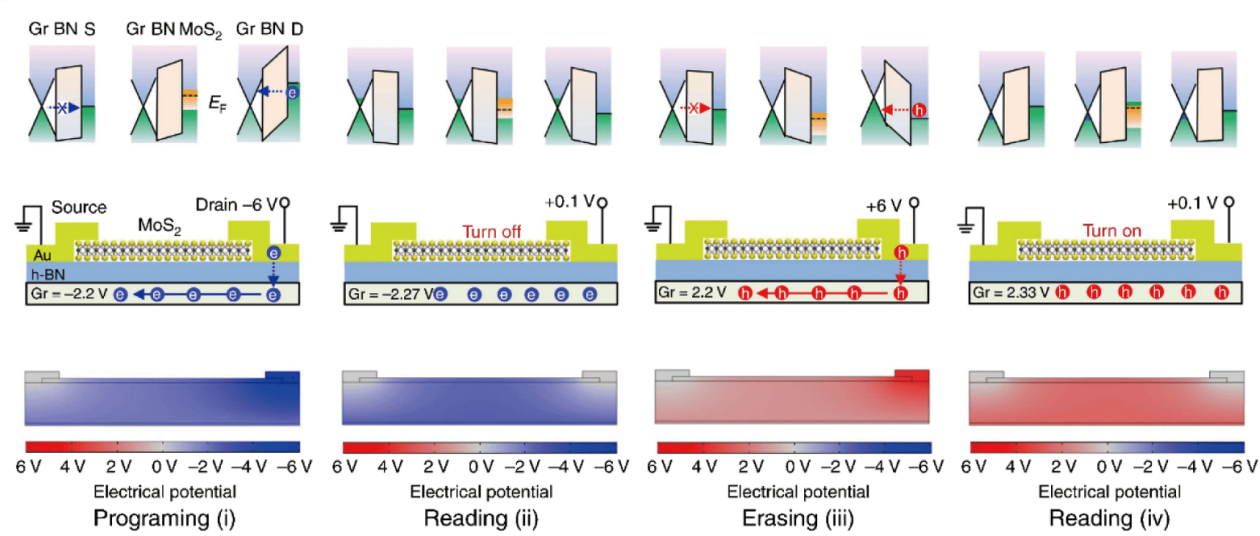

Figure 14 Two-terminal floating-gate memristor (a) device structure schematic containing an h-BN tunneling barrier, (b) band alignments during operation, (c) typical $I-V$ curve showing a current hysteresis loop, and (d) memristor operation mechanisms with four states of programing, reading, erasing, and reading. Adapted with permission from Ref. [167], Copyright 2016, Springer Nature. 
transport. As shown in Fig. 15a, a structure of graphene/ h-BN/TMD/h-BN/graphene was fabricated [176]. Under a bias voltage above a certain threshold, the electrons and holes are injected into the middle layer of the TMD from each graphene electrode. Because the quasiparticles in the quantum wells have a longer lifetime, the electrons and holes are recombined, emitting photons. As desired, the wavelength of emitted light can be precisely adjusted by changing the materials. The external quantum efficiency (EQE) in an LED based on a single quantum well is $>1 \%$. This EQE can be largely improved by combining multiple quantum wells together, resulting in a value of $8.4 \%$ in a device containing four quantum wells. Compared with $\mathrm{MoX}_{2}$ materials, such as $\mathrm{MoS}_{2}$ or $\mathrm{MoTe}_{2}$, WSe 2 is a more active light-emitting material that obtains a room-temperature $\mathrm{EQE}$ of $5 \%$ in a single quantum well structure [177]. LEDs achieved from 2D quantum wells present excellent performance that is comparable to traditional organic LEDs, highlighting their promise for future development as components in flexible optoelectronics.

\section{Photodetectors}

Generally, photodetection using 2D vdW heterostructures is realized via a phototransistor, photoconductor, photovoltaic p-n junction device, or photothermoelectric device [178-180]. Among these, devices based on the quantum tunneling mechanism possess a much faster speed than that of conventional carrier drift. In Fig. 15b, a typical photodetector was developed from a tunnel diode with a type III heterostructure and demonstrated a distinct working mechanism as a type II photodiode [124]. In type II p-n junctions, holes and electrons are depleted near the heterointerface. In such a situation, the photocurrent in $I-V$ characterization shows a shift towards the fourth quadrant. In type III tunnel photodiodes, the carriers of opposite sign in the $\mathrm{BP} / \mathrm{SnSe}_{2}$ heterojunction accumulate near the heterointerface because of broken band alignment. As shown in Fig. 15c, this leads to a shift of photoresponse curves to the second quadrant with a negative open-circuit voltage $\left(V_{o c}\right)$. Upon illumination with a 488-nm laser, the photoresponsivity at zero bias is estimated to be $\sim 0.24 \mathrm{~mA} \mathrm{~W}^{-1}$. Replacing the $\mathrm{SnSe}_{2}$ with the high-absorbance-coefficient material $\mathrm{ReS}_{2}$, the $\mathrm{BP} / \mathrm{ReS}_{2}$ heterojunction shows a photoresponsivity of $8 \mathrm{~mA} \mathrm{~W}^{-1}$ with a laser irradiation of $532 \mathrm{~nm}$ [181]. Photovoltaic energy conversion is probed with a peak EQE of $0.3 \%$. By adopting the narrow bandgap material of $\mathrm{MoTe}_{2}$, the device is able to work in the infrared (IR) region with a high photodetectivity of $7.5 \times 10^{12}$ Jones under an $811-\mathrm{nm}$ laser [182]. The photovoltaic effect is clearly observed in both the second and fourth quadrants at different gate voltages because of the ambipolar properties of $\mathrm{MoTe}_{2}$ in its transfer curves.

In addition to material choice to improve photodetection performance, photodetection can be enhanced with
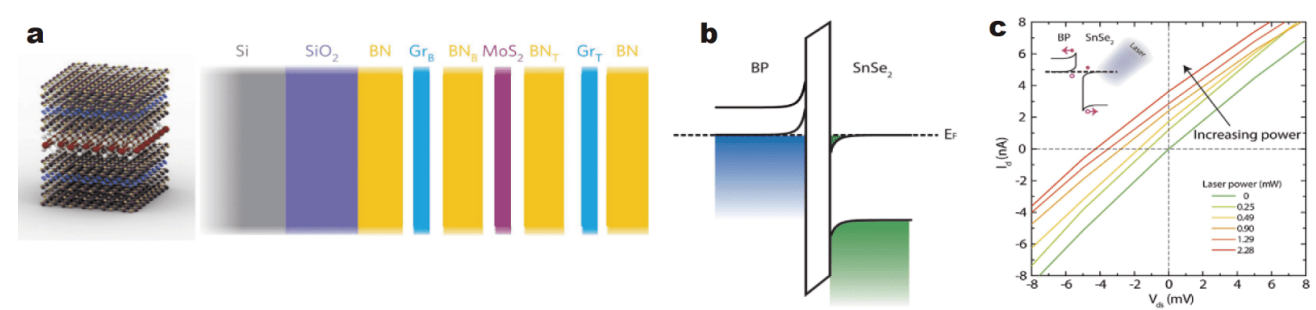

d

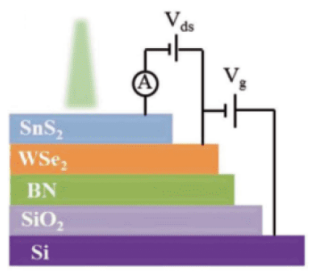

e

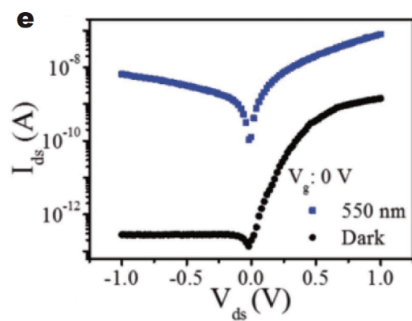

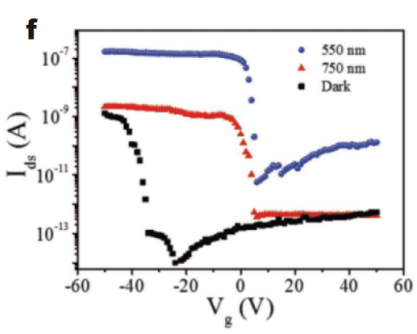

Figure 15 Optoelectrical devices based on quantum tunneling. (a) Light-emitting device with the $\mathrm{Gr}_{\mathrm{B}} / \mathrm{h}-\mathrm{BN}_{\mathrm{B}} / \mathrm{MoS}_{2} / \mathrm{h}-\mathrm{BN}_{\mathrm{T}} / \mathrm{Gr}_{\mathrm{T}}$ heterostructure, where the $\mathrm{MoS}_{2}$ receives the electrons and holes injected from two graphene layers by quantum tunneling. Adapted with permission from Ref. [176], Copyright 2015, Springer Nature. Tunnel photodiode (b) device architecture using BP/SnSe $e_{2}$ heterostructure and (c) negative open-circuit voltage in the photoresponse. (b, c) Adapted with permission from Ref. [124], Copyright 2015, American Chemical Society. Tunnel photodetector (d) device architecture using an h-BN substrate, (e) photoresponse as a diode and (f) photoresponse as an FET. (d-f) Adapted with permission from Ref. [183], Copyright 2018, Wiley. 
structural engineering. With an h-BN substrate, interlayer coupling in a $\mathrm{WSe}_{2} / \mathrm{SnS}_{2}$ junction is reinforced, enabling high tunneling current and suppressed dark current in the devices shown in Fig. 15d-f [183]. The tunnel photodetector produces an ultrahigh photodetectivity of 1.29 $\times 10^{13}$ Jones and high responsivity of $244 \mathrm{~A} \mathrm{~W}^{-1}$ while exposed to a 550-nm laser, in addition to broad photosensitivity from the ultraviolet (UV) to IR spectrum. In addition to the h-BN substrate, the post-annealing process, shown in Fig. 16a-c, is an effective method to strengthen interfacial coupling [184]. This results in band-to-band tunneling of electrons, which induces a positive photocurrent in the $\mathrm{MoS}_{2} / \mathrm{MoSe}_{2}$ heterostructure. However, only enhancing the interlayer coupling is a distant approach to high photodetector performance; additional innovative architectures are desirable for specific targets.

One of the important parameters in photodetection is the detectable wavelength region. An efficient approach to broaden it is employing gapless graphene as the widespectral-absorbance material. In this device design, fast electron-hole pair separation architecture should be introduced to prevent recombination [185]. Based on these fundamentals, a photodetector based on the $\mathrm{MoS}_{2}$ /graphene/WSe $\mathrm{W}_{2}$ heterostructure was designed and is illustrated in Fig. 16d [186]. When the irradiation light has an energy over the bandgap of $\mathrm{MoS}_{2}$ and $\mathrm{WSe}_{2}$, all three materials serve as an efficient absorbance layer, generating a photocurrent. While with an irradiation energy lower than the bandgap of $\mathrm{MoS}_{2}$ and $\mathrm{WSe}_{2}$, only graphene can absorb the photons, extending the detection spectrum from visible to the near IR region, as shown in Fig. 16e, $\mathrm{f}$. Driven by the built-in electrical field, the photogenerated carriers are rapidly separated by interlayer tunneling, endowing the device with fast detection and high gain, responsivity, and detectivity.

Another primary parameter in the design is photodetectivity, which is dramatically confined by high off current in the dark. To overcome this limitation, an ultrasensitive photodetector with a $\mathrm{MoS}_{2} / \mathrm{h}$ - $\mathrm{BN} /$ graphene heterostructure is inspired (Fig. 17a) where the graphene serves as the electrode and $\mathrm{MoS}_{2}$ is the photon absorbance layer [187]. By inserting an h-BN tunneling barrier, the dark current is largely reduced by the high energy barrier at the graphene/h-BN junction, while the photogenerated carriers can tunnel across the low barrier at the $\mathrm{MoS}_{2} / \mathrm{h}$ BN junction. At an optimized h-BN thickness of $7 \mathrm{~nm}$, a high photocurrent and low dark current are achieved with an on/off ratio larger than $10^{5}$, as well as a high detectivity of $2.6 \times 10^{13}$ Jones and responsivity of $180 \mathrm{~A} \mathrm{~W}^{-1}$, as shown in Fig. 17b, c, respectively. In addition to h-BN, $\mathrm{MoS}_{2}$ can be adopted as the tunneling

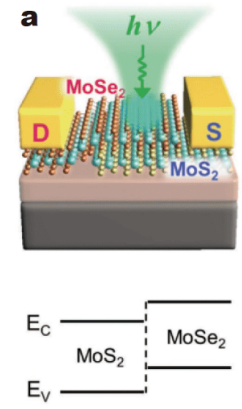

d

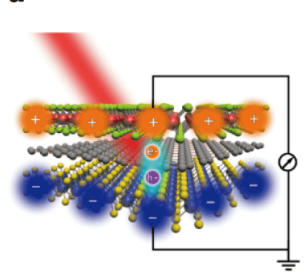

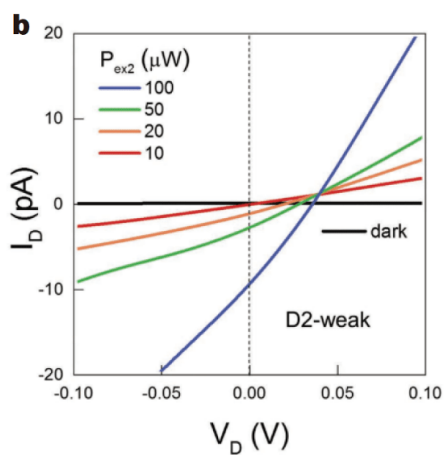

e

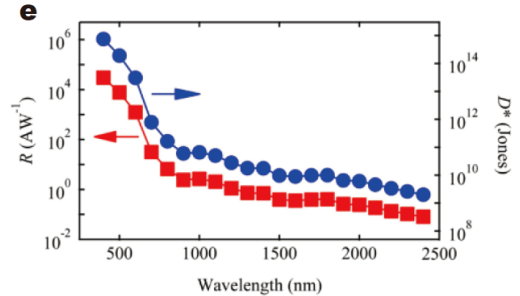

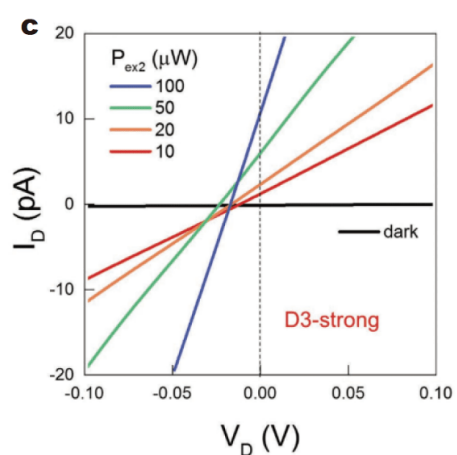
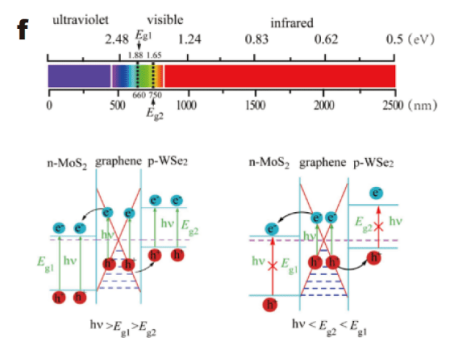

Figure 16 Tunnel photodiode (a) device structure and band alignment, (b) photoresponse in a non-annealing device, and (c) improved photoresponse in a post-annealed device. (a-c) Adapted with permission from Ref. [184], Copyright 2017, Wiley. (d) Schematic of a broadband photodetector based on the $\mathrm{MoS}_{2}$ /graphene/WSe 2 heterostructure, (e) photoresponsivity, detectivity, and (f) broadband photoresponse mechanism. (d-f) Adapted with permission from Ref. [186], Copyright 2016, American Chemical Society. 


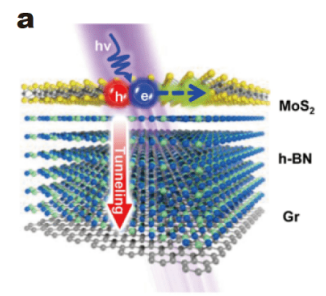

d
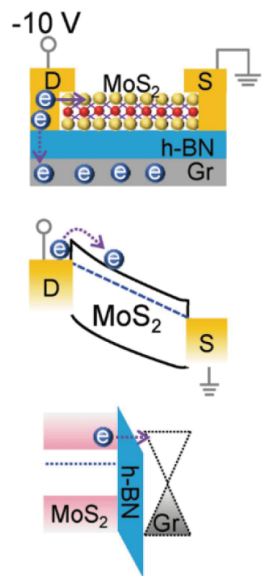

(1) Programming
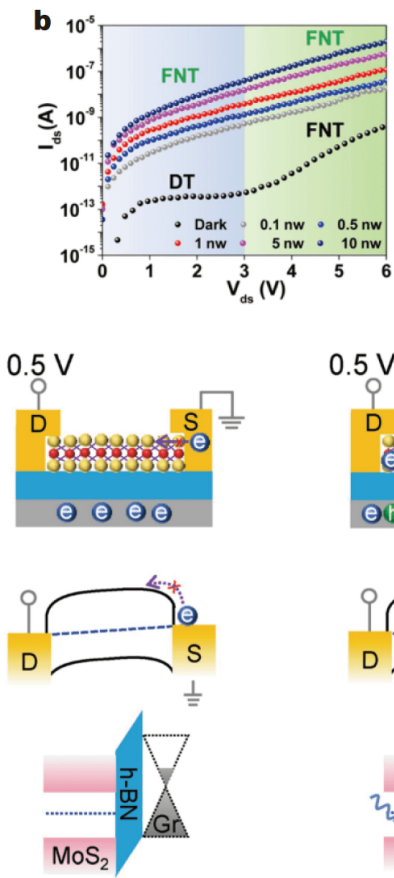

(2) Off-current reading
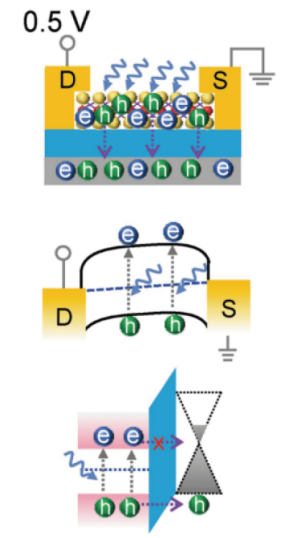

(3) Erasing
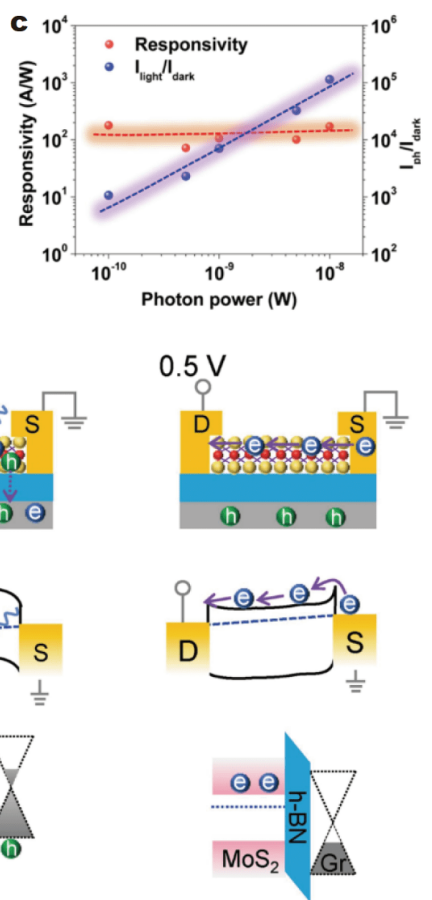

(4) On-current reading

Figure 17 Tunnel photodiode with (a) device structure using an h-BN barrier, (b) photoresponse fitted by different tunneling mechanisms, and (c) photoresponsivity as a function of photon power. (a-c) Adapted with permission from Ref. [187], Copyright 2017, American Chemical Society. (d) Two-terminal multilevel optical memory based on the $\mathrm{MoS}_{2} / \mathrm{h}$ - $\mathrm{BN} /$ graphene heterostructure, allowing programming, off-current reading, erasing, and on-current reading. Adapted with permission from Ref. [198], Copyright 2018, Wiley.

barrier, forming a heterostructure of graphene/ $\mathrm{MoS}_{2} / \mathrm{Si}$ [188]. The photogenerated carriers in Si are rapidly transferred into the graphene via the quantum tunneling process, enabling fast response and high responsibility.

\section{Optical memory applications}

2D materials and their vdW heterostructures are considered an intriguing platform to examine nonvolatile optical memory because of strong light-matter interactions [189-191]. With an ultra-large surface-to-volume ratio, $2 \mathrm{D}$ materials exhibit strong trapping of photogenerated carriers, which is frequently observed as photogating effects [192-194]. The realization of nonvolatile optical memory has been reported using a $\mathrm{CuIn}_{7} \mathrm{Se}_{11^{-}}$ based FET [195], as well as by adopting a single layer of $\mathrm{MoS}_{2}$ [196] and a hybrid of $\mathrm{Gr} / \mathrm{MoS}_{2}$ [197]. However, these devices show relatively poor performance with high dark currents, small optical switching on/off ratios, and short retention times. In terms of developing $2 \mathrm{D}$ storage electrics with high quality, such devices are extremely confined in efficiency and integration density. Alternatively, a two-terminal multilevel optical memory is proposed based on the floating-gate memory device. A
$\mathrm{MoS}_{2} / \mathrm{h}-\mathrm{BN} /$ graphene heterostructure is shown in Fig. 17d [198], wherein the top monolayer $\mathrm{MoS}_{2}$ works as the transport channel and light absorbance layer, and the bottom graphene is the floating gate. Driven by a negative drain bias pulse, electrons from $\mathrm{MoS}_{2}$ tunnel into graphene for storing, acting as the negative back gate that turns off $\mathrm{MoS}_{2}$. Under light pulse irradiation, the photogenerated holes in $\mathrm{MoS}_{2}$ are able to tunnel into graphene via FN tunneling, neutralizing the stored electrons in graphene. With the advantage of the h-BN tunneling barrier, the device shows an ultra-low dark current level at $10^{-14} \mathrm{~A}$ and a high optical on/off ratio exceeding $10^{6}$. A program-erase cycle can be endured $10^{4}$ times with a retention time longer than $3.6 \times 10^{4} \mathrm{~s}$. Eighteen distinct current levels in total are presented in measurements, corresponding to more than four bits (four current levels of one bit) storage capability.

\section{Magnetic devices}

Recently, in addition to electronic and optoelectronic properties in $2 \mathrm{D}$ materials, the magneto-electrical response of $2 \mathrm{D}$ materials has attracted attention. Among the potential applications, MTJs are the fundamental 
building blocks for magnetic sensing and data storage techniques [199]. Thus, the study of TMR devices based on $\mathrm{vdW}$ heterostructures is of great interest. Because of the progress in investigations of 2D-layered ferromagnetic materials, the development of their applications, such as spin valves, spin filters, and spin-transfer torques, has been boosted.

In general, magnetic tunneling devices rely on tunable magnetization direction in an individual ferromagnet. Monolayer $\mathrm{CrI}_{3}$ has been reported to be an Ising ferromagnetic material with a Curie temperature at $45 \mathrm{~K}$ and perpendicular magnetic anisotropy (PMA) [173]. The emerging $\mathrm{CrI}_{3}$ and its analog of $\mathrm{CrBr}_{3}$ enrich the family of magnetic insulators, which are a key resource to exploiting next-generation spintronics [200,201,202]. Furthermore, $\mathrm{MnPS}_{3}$ is a newly emerged magnetic $2 \mathrm{D}$ material that has attracted wide attention [203,204]. An atomically thin $\mathrm{CrI}_{3}$ is considered as the tunneling barrier in Fig. 18a; the tunneling conductance is probed from two contacts of graphite at various temperatures and magnetic fields. The observed magnetoresistance change is $95 \%$ and $550 \%$ in the bilayer and four-layer structures in Fig. 18b, c, respectively [205]. At a strong magnetic field of $2 \mathrm{~T}$, a very large magnetoresistance of one million percent is achieved at low temperatures [206]. This magnetoresistance is derived from the variation between antiparallel and parallel alignment of spin carriers in each $\mathrm{CrI}_{3}$ layer. The $\mathrm{CrI}_{3}$ is characterized to have intrinsic layer-by-layer anti-ferromagnetic ordering. Based on these discoveries, a spin filter MTJ with the vdW heterostructure of graphene/ $\mathrm{CrI}_{3} /$ graphene has been reported [207]. The tunneling magnetoresistance is dramatically increased with a thicker $\mathrm{CrI}_{3}$ layer, achieving a high record of $19,000 \%$ in a four-layer structure at low temperature. This device structure is classified as the conductor/insulator/conductor structure, and the ferromagnetic properties are detected from the insulating layer. Another type of spin valve with an opposite structure was proposed, wherein the $2 \mathrm{D}$ nonmagnetic conductor is sandwiched between two ferromagnetic insulating layers [208]. Consequently, the relative magnetization directions in each insulator strongly affect the inplane conductivity in the conductor. By constructing a $\mathrm{vdW}$ heterostructure where the bilayer graphene is surrounded by two monolayers of ferromagnetic insulator $\mathrm{CrI}_{3}$, the bandgap opening phenomenon at the Dirac point is predicted in the antiparallel case.

$\mathrm{Fe}_{3} \mathrm{GeTe}_{2}$ was observed to have robust ferromagnetism, even in the monolayer, with a Curie temperature at $130 \mathrm{~K}$ [209]. By adopting an ionic gate, the Curie temperature can be increased to room temperature [210]. Fig. $18 \mathrm{~d}-\mathrm{f}$ show the realization and investigation of $\mathrm{Fe}_{3} \mathrm{GeTe}_{2}$-based tunneling spin valves [211]. The vdW heterostructure contains a thin h-BN layer as the tunneling barrier, which is sandwiched between two ferromagnetic $\mathrm{Fe}_{3} \mathrm{GeTe}_{2}$ electrodes. When the magnetization directions in the two

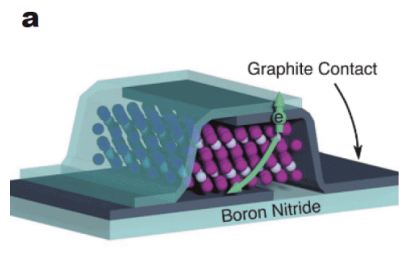

d

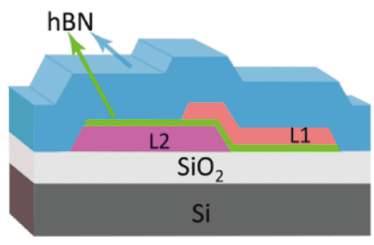

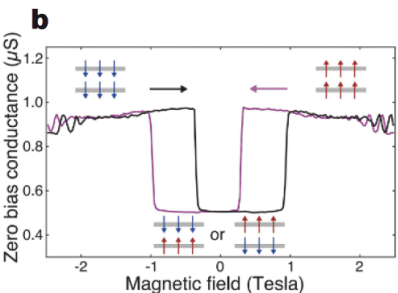

e

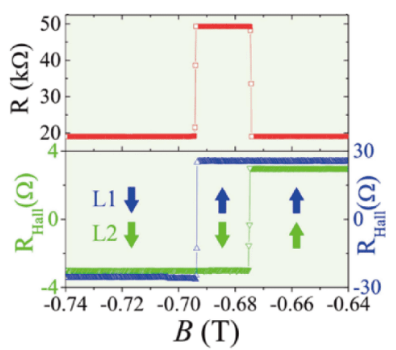

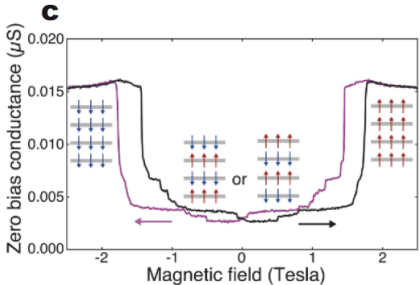

$\mathbf{f}$

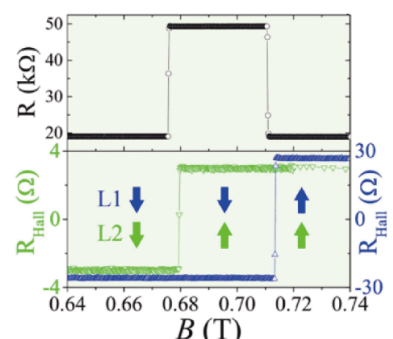

Figure 18 MTJs. (a) Structural schematic and conductance through (b) a bilayer $\mathrm{CrI}_{3}$ and (c) a four-layer $\mathrm{CrI}_{3}$ as a function of the out-of-plane magnetic field for a device based on the graphene/ $\mathrm{CrI}_{3} /$ graphene heterostructure. (a-c) Adapted with permission from Ref. [205], Copyright 2018, the American Association for the Advancement of Science. (d) Structural schematic, spin-valve effect at (e) negative and (f) positive magnetic field range, respectively, for a device based on the $\mathrm{Fe}_{3} \mathrm{GeTe}_{2} / \mathrm{h}-\mathrm{BN} / \mathrm{Fe}_{3} \mathrm{GeTe}_{2}$ heterostructure. (d-f) Adapted with permission from Ref. [211], Copyright 2018, American Chemical Society. 
electrodes are aligned in parallel to each other, the tunneling resistance reaches a minimum, which turns to a maximum when the directions are antiparallel. The magnetoresistance change is measured to be $160 \%$ at low temperatures. The resultant spin polarization of $\mathrm{Fe}_{3} \mathrm{GeTe}_{2}$ is 0.66 , representing an $83 \%$ majority with $17 \%$ minority spin carriers.

Traditional TMD materials have magnetic properties. Spin-polarized tunneling across a multilayer of $\mathrm{MoS}_{2}$ was reported at room temperature using a $\mathrm{Co} / \mathrm{MoS}_{2} / \mathrm{Ni}_{80} \mathrm{Fe}_{20}$ structure [212]. This vertical spin-valve shows a TMR of $0.5 \%-2 \%$, corresponding to a spin polarization of $5 \%-$ $10 \%$ at temperatures ranging from 75 to $300 \mathrm{~K}$. With this intriguing result, a theoretical prediction of a vdW MTJ based on the $\mathrm{VSe}_{2} / \mathrm{MoS}_{2} / \mathrm{VSe}_{2}$ heterojunction was presented [213], wherein the monolayer $\mathrm{VSe}_{2}$ was characterized to be an intrinsic ferromagnetic layer at room temperature [214]. Because of the strong spin Hall conductivity of $\mathrm{MoS}_{2}$, a large TMR of $846 \%$ is predicted at $300 \mathrm{~K}$. These discoveries advance a platform to exploit spintronics in $2 \mathrm{D}$ semiconductors and magnetic insulators.

\section{CONCLUSION AND CHALLENGES}

To date, 2D materials and their vdW heterostructures have amazed the world in diverse fields, from the basic research of quantum physics to industrial applications for high-density integration. They offer a superior platform to examine low-dimensional electronics, optics, and magnetics [215-217]. Among their applications, tunneling devices with high performance have drawn attention because of their development, opportunities, as well as challenges. Unlike 3D junctions, 2D materials are atomically thin and free of band bending. The band alignment in vertical $2 \mathrm{D}$ junctions lacks a depletion region, promoting quantum tunneling with an extremely narrow barrier. In this review, we primarily focused on the formation, properties, and applications of quantum tunneling across vdW heterostructures. In this stage, the expression of quantum tunneling in $2 \mathrm{D}$ vdW heterostructures is no longer a difficult task. Despite the extraordinary progress to date, there are still challenges, a list of which follows, that remain to be tackled.

\section{Device quality}

One of the foremost concerns is the control of device quality during material preparation and construction of $\mathrm{vdW}$ heterostructures to further practical integration, which significantly affects quantum tunneling performance. Because of pioneering efforts in recent years, re- searchers now can fabricate $2 \mathrm{D}$ vdW heterostructures with any desired function [218,219]. In general, the heterostructures can be layered by physical stacking or CVD. The physical method, however, always struggles with the presence of interfacial contamination. During the transfer stacking process, impurities from ambient air stick on material surfaces, forming bubbles (referred to as blisters or pockets) at the interfaces [220,221]. Even these adsorbates can be controlled by the self-cleansing effect such that they are driven and moved along the interfaces; however, interlayer bubbles are still completely irretrievable. These bubbles act as trapping centers, inducing recombination of carriers with seriously degraded tunneling properties. The heating-assisted transfer method can efficiently suppress the formation of bubbles by driving adsorbates outside of the overlapped regions; however, there are still challenges to achieving the ideal clean interface. In terms of the chemical approach, it is first greatly limited by material choices when three or more materials are required. Furthermore, the compatibility of synthesis environments with different materials must be strictly considered when using CVD techniques.

\section{Device scale}

Although the electrical performance of CVD-grown samples is inferior to that of exfoliated samples, largescale films are the viable solution for integrating highdensity logic circuits. As for the usage of graphene, the roll-to-roll method can offer large-scale transfer with reliable quality. Nevertheless, when other $2 \mathrm{D}$ materials are adopted, such as the frequently used tunneling barrier of h-BN, their practical full film transfer techniques or direct syntheses of various vdW heterostructures have not been fully explored.

\section{Device arbitrariness}

Compared with the physical approach, CVD production is much less arbitrary. The transfer construction of largescale samples lacks tunability of the relative twist angles between the adjacent layers, which is an important factor for determining resonant quantum tunneling. Great manual effort is always required to access precise deposition at appointed positions. Furthermore, highly selective etching techniques to isolate building blocks and curve individual devices have been rarely reported and are in extreme requirement of further study.

\section{Device contact}

Another challenge concerns contact issues for practical applications. The atomically thin geometry of $2 \mathrm{D}$ mate- 
rials causes the quantum tunneling mechanism to be slightly different from that in 3D devices, whereby the contact with the transport channel plays an important role that should be addressed during device design. In addition to quantum tunneling current, thermionic emission simultaneously exists at room temperature. In such a situation, a large thermionic emission current would overwhelm quantum tunneling, shadowing the intrinsic properties of the materials. To develop an independent and reliable contact with each complex vdW heterostructure, a novel contact scheme should be developed to bring this field one step forward.

Overall, there is still considerable scope for gaining a complete understanding of 2D tunneling devices. Emerging new materials, new vdW heterostructures, and new device functions would provide exceptional opportunities and inspire potential targeting in future advanced electronics.

Received 6 January 2021; accepted 15 April 2021;

published online 28 June 2021

1 Grifoni M, Hänggi P. Driven quantum tunneling. Phys Rep, 1998, 304: $229-354$

2 Herring C, Nichols MH. Thermionic emission. Rev Mod Phys, 1949, 21: 185-270

3 Hara K, Ohba I. Tunneling time distribution by means of Nelson's quantum mechanics and wave-particle duality. Phys Rev A, 2003, 67: 052105

4 Wang $\mathrm{CH}$, Hong TM, Lee RK, et al. Particle-wave duality in quantum tunneling of a bright soliton. Opt Express, 2012, 20: 22675

5 Chen JF, Yang L, Wu MC, et al. On the effect of the barrier widths in the InAs/AlSb/GaSb single-barrier interband tunneling structures. J Appl Phys, 1990, 68: 3451-3455

6 Ionescu AM, Riel H. Tunnel field-effect transistors as energyefficient electronic switches. Nature, 2011, 479: 329-337

7 Verhulst AS, Vandenberghe WG, Maex K, et al. Tunnel fieldeffect transistor without gate-drain overlap. Appl Phys Lett, 2007, 91: 053102

8 Bhuwalka KK, Sedlmaier S, Ludsteck AK, et al. Vertical tunnel field-effect transistor. IEEE Trans Electron Devices, 2004, 51: 279-282

9 Cao XA, Stokes EB, Sandvik PM, et al. Diffusion and tunneling currents in $\mathrm{GaN} / \mathrm{InGaN}$ multiple quantum well light-emitting diodes. IEEE Electron Device Lett, 2002, 23: 535-537

10 Lin GR, Lin CJ, Lin CK. Enhanced Fowler-Nordheim tunneling effect in nanocrystallite Si based LED with interfacial Si nanopyramids. Opt Express, 2007, 15: 2555-2563

11 Parzefall M, Szabó Á, Taniguchi T, et al. Light from van der Waals quantum tunneling devices. Nat Commun, 2019, 10: 292

12 O'Brien JL. Optical quantum computing. Science, 2007, 318: 1567-1570

13 Walther P, Resch KJ, Rudolph T, et al. Experimental one-way quantum computing. Nature, 2005, 434: 169-176

14 Leuenberger MN, Loss D. Quantum computing in molecular magnets. Nature, 2001, 410: 789-793

15 Gatteschi D, Sessoli R. Quantum tunneling of magnetization and related phenomena in molecular materials. Angew Chem Int Ed, 2003, 42: 268-297

16 Chudnovsky EM, Gunther L. Quantum tunneling of magnetization in small ferromagnetic particles. Phys Rev Lett, 1988, 60: 661-664

17 Sangregorio C, Ohm T, Paulsen C, et al. Quantum tunneling of the magnetization in an iron cluster nanomagnet. Phys Rev Lett, 1997, 78: 4645-4648

18 Binnig G, Rohrer H. Scanning tunneling microscopy. Surf Sci, 1983, 126: 236-244

19 Binning G, Rohrer H, Gerber C, et al. Surface studies by scanning tunneling microscopy. Phys Rev Lett, 1982, 49: 57-61

20 Zhao M, Kim D, Nguyen VL, et al. Coherent thermoelectric power from graphene quantum dots. Nano Lett, 2019, 19: 61-68

21 Moore GE. Progress in digital integrated electronics. In: Proceedings of International Electron Devices Meeting. Washington: IEEE, 1975, 11-13

22 Radisavljevic B, Radenovic A, Brivio J, et al. Single-layer $\mathrm{MoS}_{2}$ transistors. Nat Nanotech, 2011, 6: 147-150

23 Desai SB, Madhvapathy SR, Sachid AB, et al. $\mathrm{MoS}_{2}$ transistors with 1-nanometer gate lengths. Science, 2016, 354: 99-102

24 Wu Y, Lin YM, Bol AA, et al. High-frequency, scaled graphene transistors on diamond-like carbon. Nature, 2011, 472: 74-78

25 Jacobsen RS, Andersen KN, Borel PI, et al. Strained silicon as a new electro-optic material. Nature, 2006, 441: 199-202

26 Thompson SE, Armstrong M, Auth C, et al. A 90-nm logic technology featuring strained-silicon. IEEE Trans Electron Devices, 2004, 51: 1790-1797

27 Higashiwaki M, Mimura T, Matsui T. High-performance shortgate InAlN/GaN heterostructure field-effect transistors. Jpn J Appl Phys, 2006, 45: L843-L845

28 Poiroux T, Vinet M, Faynot $\mathrm{O}$, et al. Multiple gate devices: Advantages and challenges. MicroElectron Eng, 2005, 80: 378-385

29 Iannaccone G, Bonaccorso F, Colombo L, et al. Quantum engineering of transistors based on 2D materials heterostructures. Nat Nanotech, 2018, 13: 183-191

30 Bao X, Ou Q, Xu ZQ, et al. Band structure engineering in $2 \mathrm{D}$ materials for optoelectronic applications. Adv Mater Technol, 2018, 3: 1800072

31 Forsythe C, Zhou X, Watanabe $\mathrm{K}$, et al. Band structure engineering of $2 \mathrm{D}$ materials using patterned dielectric superlattices. Nat Nanotech, 2018, 13: 566-571

32 Sun Y, Zhou Z, Huang Z, et al. Band structure engineering of interfacial semiconductors based on atomically thin lead iodide crystals. Adv Mater, 2019, 31: 1806562

33 Kim K. From the future Si technology perspective: Challenges and opportunities. In: Proceedings of 2010 International Electron Devices Meeting. San Francisco: IEEE, 2010, 1-9

34 Paul DJ, See P, Zozoulenko IV, et al. n-Type Si/SiGe resonant tunnelling diodes. Mater Sci Eng-B, 2002, 89: 26-29

35 Liao Y, Zhang P, Bremner S, et al. Resonant tunneling through monolayer Si colloidal quantum dots and Ge nanocrystals. Adv Funct Mater, 2017, 27: 1605348

36 Khayer MA, Lake RK. Effects of band-tails on the subthreshold characteristics of nanowire band-to-band tunneling transistors. J Appl Phys, 2011, 110: 074508

37 Huisman SR, Ctistis G, Stobbe S, et al. Measurement of a bandedge tail in the density of states of a photonic-crystal waveguide. 
Phys Rev B, 2012, 86: 155154

38 Kirchartz T, Pieters BE, Kirkpatrick J, et al. Recombination via tail states in polythiophene: Fullerene solar cells. Phys Rev B, 2011, 83: 115209

39 Kargar A, Sukrittanon S, Zhou C, et al. GaP/GaNP heterojunctions for efficient solar-driven water oxidation. Small, 2017, 13: 1603574

40 Lee CH, Krishnamoorthy S, Paul PK, et al. Large-area $\mathrm{SnSe}_{2} / \mathrm{GaN}$ heterojunction diodes grown by molecular beam epitaxy. Appl Phys Lett, 2017, 111: 202101

41 Ramesh A, Berger PR, Loo R. High 5.2 peak-to-valley current ratio in $\mathrm{Si} / \mathrm{SiGe}$ resonant interband tunnel diodes grown by chemical vapor deposition. Appl Phys Lett, 2012, 100: 092104

42 Alshehri AH, Mistry K, Nguyen VH, et al. Quantum-tunneling metal-insulator-metal diodes made by rapid atmospheric pressure chemical vapor deposition. Adv Funct Mater, 2019, 29: 1805533

43 Levine BF. Quantum-well infrared photodetectors. J Appl Phys, 1993, 74: R1-R81

44 Cheng L, Jiang T, Cao Y, et al. Multiple-quantum-well perovskites for high-performance light-emitting diodes. Adv Mater, 2020, 32: 1904163

45 Novoselov KS, Mishchenko A, Carvalho A, et al. 2D materials and van der Waals heterostructures. Science, 2016, 353: aac9439

46 Geim AK, Grigorieva IV. van der Waals heterostructures. Nature, 2013, 499: 419-425

47 Gupta A, Sakthivel T, Seal S. Recent development in 2D materials beyond graphene. Prog Mater Sci, 2015, 73: 44-126

48 Jariwala D, Marks TJ, Hersam MC. Mixed-dimensional van der Waals heterostructures. Nat Mater, 2017, 16: 170-181

49 Kim KK, Lee HS, Lee YH. Synthesis of hexagonal boron nitride heterostructures for 2D van der Waals electronics. Chem Soc Rev, 2018, 47: 6342-6369

50 Jin C, Ma EY, Karni O, et al. Ultrafast dynamics in van der Waals heterostructures. Nat Nanotech, 2018, 13: 994-1003

51 Gibertini M, Koperski M, Morpurgo AF, et al. Magnetic 2D materials and heterostructures. Nat Nanotechnol, 2019, 14: 408419

52 Li H, Ruan S, Zeng YJ. Intrinsic van der Waals magnetic materials from bulk to the 2D limit: New frontiers of spintronics. Adv Mater, 2019, 31: 1900065

53 Liu L, Feng YP, Shen ZX. Structural and electronic properties of h-BN. Phys Rev B, 2003, 68: 104102

54 Wang L, Chen Z, Dean CR, et al. Negligible environmental sensitivity of graphene in a hexagonal boron nitride/graphene/hBN sandwich structure. ACS Nano, 2012, 6: 9314-9319

55 Furchi MM, Pospischil A, Libisch F, et al. Photovoltaic effect in an electrically tunable van der Waals heterojunction. Nano Lett, 2014, 14: 4785-4791

56 Hong X, Kim J, Shi SF, et al. Ultrafast charge transfer in atomically thin $\mathrm{MoS}_{2} / \mathrm{WS}_{2}$ heterostructures. Nat Nanotech, 2014, 9: 682-686

57 Cheng R, Li D, Zhou H, et al. Electroluminescence and photocurrent generation from atomically sharp $\mathrm{WSe}_{2} / \mathrm{MoS}_{2}$ heterojunction p-n diodes. Nano Lett, 2014, 14: 5590-5597

58 Li L, Yu Y, Ye GJ, et al. Black phosphorus field-effect transistors. Nat Nanotech, 2014, 9: 372-377

59 Abbas AN, Liu B, Chen L, et al. Black phosphorus gas sensors. ACS Nano, 2015, 9: 5618-5624

60 Jiang X, Zhao X, Bao W, et al. Graphdiyne nanosheets for multicolor random lasers. ACS Appl Nano Mater, 2020, 3: 4990-4996
61 Wu L, Dong Y, Zhao J, et al. Kerr nonlinearity in 2D graphdiyne for passive photonic diodes. Adv Mater, 2019, 31: 1807981

$62 \mathrm{Wu} \mathrm{W}$, Qiu G, Wang Y, et al. Tellurene: Its physical properties, scalable nanomanufacturing, and device applications. Chem Soc Rev, 2018, 47: 7203-7212

63 Wang Y, Qiu G, Wang R, et al. Field-effect transistors made from solution-grown two-dimensional tellurene. Nat Electron, 2018, 1: 228-236

64 Wang $\mathrm{C}$, Wang $\mathrm{Y}$, Jiang $\mathrm{X}$, et al. MXene $\mathrm{Ti}_{3} \mathrm{C}_{2} \mathrm{~T}_{x}$ : A promising photothermal conversion material and application in all-optical modulation and all-optical information loading. Adv Opt Mater, 2019, 7: 1900060

65 Wu L, Jiang X, Zhao J, et al. MXene-based nonlinear optical information converter for all-optical modulator and switcher. Laser Photonics Rev, 2018, 12: 1800215

66 Lin YM, Valdes-Garcia A, Han SJ, et al. Wafer-scale graphene integrated circuit. Science, 2011, 332: 1294-1297

67 Shi J, Chen X, Zhao L, et al. Chemical vapor deposition grown wafer-scale $2 \mathrm{D}$ tantalum diselenide with robust charge-densitywave order. Adv Mater, 2018, 30: 1804616

68 Mattinen M, King PJ, Khriachtchev L, et al. Low-temperature wafer-scale deposition of continuous 2D SnS 2 films. Small, 2018, 14: 1800547

69 Yang Z, Jie W, Mak CH, et al. Wafer-scale synthesis of highquality semiconducting two-dimensional layered InSe with broadband photoresponse. ACS Nano, 2017, 11: 4225-4236

70 Simmons JG. Generalized formula for the electric tunnel effect between similar electrodes separated by a thin insulating film. J Appl Phys, 1963, 34: 1793-1803

71 Murphy EL, Good RH. Thermionic emission, field emission, and the transition region. Phys Rev, 1956, 102: 1464-1473

72 Müller M, Miao GX, Moodera JS. Exchange splitting and biasdependent transport in $\mathrm{EuO}$ spin filter tunnel barriers. Europhys Lett, 2009, 88: 47006

73 Lee GH, Yu YJ, Lee C, et al. Electron tunneling through atomically flat and ultrathin hexagonal boron nitride. Appl Phys Lett, 2011, 99: 243114

74 Ma Q, Andersen TI, Nair NL, et al. Tuning ultrafast electron thermalization pathways in a van der Waals heterostructure. Nat Phys, 2016, 12: 455-459

75 Esaki L. Long journey into tunneling. Science, 1974, 183: 11491155

76 Chang LL, Esaki L. Tunnel triode-a tunneling base transistor. Appl Phys Lett, 1977, 31: 687-689

77 Alekseev E, Pavlidis D. Large-signal microwave performance of GaN-based NDR diode oscillators. Solid-State Electron, 2000, 44: 941-947

78 Bilbao-Guillerna A, De la Sen M, Alonso-Quesada S, et al. A stable multimodel scheme control for the regulation of the transient behavior of a tunnel-diode trigger circuit. ISA Trans, 2007, 46: 313-326

79 Van Degrift CT. Tunnel diode oscillator for $0.001 \mathrm{ppm}$ measurements at low temperatures. Rev Sci Instruments, 1975, 46: 599-607

80 Winitzki S. Cosmological particle production and the precision of the WKB approximation. Phys Rev D, 2005, 72: 104011

81 Fan S, Vu QA, Lee $\mathrm{S}$, et al. Tunable negative differential resistance in van der Waals heterostructures at room temperature by tailoring the interface. ACS Nano, 2019, 13: 8193-8201

82 Smith JT, Das S, Appenzeller J. Broken-gap tunnel MOSFET: A 
constant-slope sub-60-mV/decade transistor. IEEE Electron Device Lett, 2011, 32: 1367-1369

83 Roy T, Tosun M, Cao X, et al. Dual-gated $\mathrm{MoS}_{2} / \mathrm{WSe}_{2}$ van der Waals tunnel diodes and transistors. ACS Nano, 2015, 9: 20712079

84 Bizindavyi J, Verhulst AS, Verreck D, et al. Large variation in temperature dependence of band-to-band tunneling current in tunnel devices. IEEE Electron Device Lett, 2019, 40: 1864-1867

85 Rideout VL. A review of the theory, technology and applications of metal-semiconductor rectifiers. Thin Solid Films, 1978, 48: 261-291

86 Salahuddin S, Datta S. Can the subthreshold swing in a classical FET be lowered below $60 \mathrm{mV} /$ decade? In: Proceedings of 2008 IEEE International Electron Devices Meeting. San Francisco: IEEE, 2008, 1-4

87 Vu QA, Fan S, Hyup Lee S, et al. Near-zero hysteresis and nearideal subthreshold swing in h-BN encapsulated single-layer $\mathrm{MoS}_{2}$ field-effect transistors. 2D Mater, 2018, 5: 031001

88 Choi WY, Park BG, Lee JD, et al. Tunneling field-effect transistors (TFETs) with subthreshold swing (SS) less than $60 \mathrm{mV} / \mathrm{dec}$. IEEE Electron Device Lett, 2007, 28: 743-745

89 Cao W, Jiang J, Kang J, et al. Designing band-to-band tunneling field-effect transistors with 2D semiconductors for next-generation low-power VLSI. In: Proceedings of 2015 IEEE International Electron Devices Meeting. Washington: IEEE, 2015, 12-3

90 Jena D. Tunneling transistors based on graphene and 2-D crystals. Proc IEEE, 2013, 101: 1585-1602

91 Gong C, Zhang H, Wang W, et al. Band alignment of two-dimensional transition metal dichalcogenides: Application in tunnel field effect transistors. Appl Phys Lett, 2013, 103: 053513

92 Kang J, Tongay S, Zhou J, et al. Band offsets and heterostructures of two-dimensional semiconductors. Appl Phys Lett, 2013, 102: 012111

93 Zhang C, Gong C, Nie Y, et al. Systematic study of electronic structure and band alignment of monolayer transition metal dichalcogenides in van der Waals heterostructures. 2D Mater, 2017, 4: 015026

94 Odoh EO, Njapba AS. A review of semiconductor quantum well devices. Adv Phys Theor Appl, 2015, 46: 26-32

95 Kuo YH, Lee YK, Ge Y, et al. Strong quantum-confined Stark effect in germanium quantum-well structures on silicon. Nature, 2005, 437: 1334-1336

96 Arakawa Y, Sakaki H. Multidimensional quantum well laser and temperature dependence of its threshold current. Appl Phys Lett, 1982, 40: 939-941

97 Tsu R, Esaki L. Tunneling in a finite superlattice. Appl Phys Lett, 1973, 22: 562-564

98 Esaki L, Tsu R. Superlattice and negative differential conductivity in semiconductors. IBM J Res Dev, 1970, 14: 61-65

99 Eisele H, Khanna SP, Linfield EH. Superlattice electronic devices as high-performance oscillators between 60-220 GHz. Appl Phys Lett, 2010, 96: 072101

100 Muhowski AJ, Ricker RJ, Boggess TF, et al. n-Type anode layer, high-power MWIR superlattice LED. Appl Phys Lett, 2017, 111: 243509

101 Kluksdahl NC, Kriman AM, Ferry DK, et al. Self-consistent study of the resonant-tunneling diode. Phys Rev B, 1989, 39: 7720-7735

102 Iogansen LV. The possibility of resonance transmission of electrons in crystals through a system of barriers. Sov Phys Jetp, 1964, 18: $146-50$
103 Soderstrom J, Andersson TG. A multiple-state memory cell based on the resonant tunneling diode. IEEE Electron Device Lett, 1988, 9: 200-202

104 Kane EO. Zener tunneling in semiconductors. J Phys Chem Solids, 1960, 12: 181-188

105 Di Carlo A, Vogl P, Pötz W. Theory of Zener tunneling and Wannier-Stark states in semiconductors. Phys Rev B, 1994, 50: 8358-8377

106 Lan C, Li C, Wang S, et al. Zener tunneling and photoresponse of a $\mathrm{WS}_{2} / \mathrm{Si}$ van der Waals heterojunction. ACS Appl Mater Interfaces, 2016, 8: 18375-18382

107 Miller SL. Avalanche breakdown in germanium. Phys Rev, 1955, 99: 1234-1241

108 Singh Tyagi M. Zener and avalanche breakdown in silicon alloyed p-n junctions-I: Analysis of reverse characteristics. Solid-State Electron, 1968, 11: 99-115

109 Moazzami R, Hu C. Stress-induced current in thin silicon dioxide films. In: Proceedings of 1992 International Technical Digest on Electron Devices Meeting. San Francisco: IEEE, 1992. 139-42

110 Riccö B, Gozzi G, Lanzoni M. Modeling and simulation of stressinduced leakage current in ultrathin $\mathrm{SiO}_{2}$ films. IEEE Trans Electron Devices, 1998, 45: 1554-1560

111 Aritome S, Shirota R, Hemink G, et al. Reliability issues of flash memory cells. Proc IEEE, 1993, 81: 776-788

112 Larcher L. Statistical simulation of leakage currents in MOS and flash memory devices with a new multiphonon trap-assisted tunneling model. IEEE Trans Electron Devices, 2003, 50: 12461253

113 Herrmann M, Schenk A. Field and high-temperature dependence of the long term charge loss in erasable programmable read only memories: Measurements and modeling. J Appl Phys, 1995, 77: 4522-4540

114 Ielmini D, Spinelli AS, Lacaita AL, et al. Modeling of anomalous SILC in flash memories based on tunneling at multiple defects. Solid-State Electron, 2002, 46: 1749-1756

115 Ielmini D, Spinelli AS, Lacaita AL, et al. Defect generation statistics in thin gate oxides. IEEE Trans Electron Devices, 2004, 51: 1288-1295

116 Schuler F, Degraeve R, Hendrickx P, Wellekens D. Physical description of anomalous charge loss in floating gate based NVM's and identification of its dominant parameter. In: Proceedings of 40th Annual International Reliability Physics Symposium. Dallas: IEEE, 2002, 26-33

117 Gould C, Rüster C, Jungwirth T, et al. Tunneling anisotropic magnetoresistance: A spin-valve-like tunnel magnetoresistance using a single magnetic layer. Phys Rev Lett, 2004, 93: 117203

118 Faure-Vincent J, Tiusan C, Jouguelet E, et al. High tunnel magnetoresistance in epitaxial $\mathrm{Fe} / \mathrm{MgO} / \mathrm{Fe}$ tunnel junctions. Appl Phys Lett, 2003, 82: 4507-4509

119 Yuasa S, Djayaprawira DD. Giant tunnel magnetoresistance in magnetic tunnel junctions with a crystalline $\mathrm{MgO}\left(\begin{array}{lll}0 & 0 & 1\end{array}\right)$ barrier. J Phys D-Appl Phys, 2007, 40: R337-R354

120 MacLaren JM, Zhang XG, Butler WH. Validity of the Julliere model of spin-dependent tunneling. Phys Rev B, 1997, 56: 1182711832

121 Qi Y, Xing DY, Dong J. Relation between Julliere and Slonczewski models of tunneling magnetoresistance. Phys Rev B, 1998, 58: 2783-2787

122 Esaki L. New phenomenon in narrow germanium $\mathrm{p}-\mathrm{n}$ junctions. Phys Rev, 1958, 109: 603-604 
123 Nourbakhsh A, Zubair A, Dresselhaus MS, et al. Transport properties of a $\mathrm{MoS}_{2} / \mathrm{WSe}_{2}$ heterojunction transistor and its potential for application. Nano Lett, 2016, 16: 1359-1366

124 Yan R, Fathipour S, Han Y, et al. Esaki diodes in van der Waals heterojunctions with broken-gap energy band alignment. Nano Lett, 2015, 15: 5791-5798

125 Shim J, Oh S, Kang DH, et al. Phosphorene/rhenium disulfide heterojunction-based negative differential resistance device for multi-valued logic. Nat Commun, 2016, 7: 13413

126 Kim KH, Park HY, Shim J, et al. A multiple negative differential resistance heterojunction device and its circuit application to ternary static random access memory. Nanoscale Horiz, 2020, 5: 654-662

127 Li MO, Esseni D, Nahas JJ, et al. Two-dimensional heterojunction interlayer tunneling field effect transistors (thin-TFETs). IEEE J Electron Devices Soc, 2015, 3: 200-207

128 Roy T, Tosun M, Hettick M, et al. 2D-2D tunneling field-effect transistors using $\mathrm{WSe}_{2} / \mathrm{SnSe}_{2}$ heterostructures. Appl Phys Lett, 2016, 108: 083111

129 Murali K, Dandu M, Das S, et al. Gate-tunable $\mathrm{WSe}_{2} / \mathrm{SnSe}_{2}$ backward diode with ultrahigh-reverse rectification ratio. ACS Appl Mater Interfaces, 2018, 10: 5657-5664

130 Wang F, Luo $\mathrm{P}$, Zhang $\mathrm{Y}$, et al. Band structure engineered tunneling heterostructures for high-performance visible and nearinfrared photodetection. Sci China Mater, 2020, 63: 1537-1547

131 Goto E, Murata K, Nakazawa K, et al. Esaki diode high-speed logical circuits. IEEE Trans Electron Comput, 1960, EC-9: 25-29

132 Yan X, Liu C, Li C, et al. Tunable $\mathrm{SnSe}_{2} / \mathrm{WSe}_{2}$ heterostructure tunneling field effect transistor. Small, 2017, 13: 1701478

133 Wang Y, Yang B, Wan B, et al. Degradation of black phosphorus: A real-time ${ }^{31}$ P NMR study. 2D Mater, 2016, 3: 035025

134 Lü JT, Cao JC. Terahertz generation and chaotic dynamics in GaN NDR diode. Semicond Sci Technol, 2004, 19: 451-456

135 Duong NT, Bang S, Lee SM, et al. Parameter control for enhanced peak-to-valley current ratio in a $\mathrm{MoS}_{2} / \mathrm{MoTe}_{2}$ van der Waals heterostructure. Nanoscale, 2018, 10: 12322-12329

136 Mahajan M, Majumdar K. Gate- and light-tunable negative differential resistance with high peak current density in $1 \mathrm{~T}-\mathrm{TaS}_{2} / 2 \mathrm{H}$ $\mathrm{MoS}_{2}$ T-junction. ACS Nano, 2020, 14: 6803-6811

137 Liu X, Qu D, Li HM, et al. Modulation of quantum tunneling via a vertical two-dimensional black phosphorus and molybdenum disulfide p-n junction. ACS Nano, 2017, 11: 9143-9150

138 Duong NT, Lee J, Bang S, et al. Modulating the functions of $\mathrm{MoS}_{2} / \mathrm{MoTe}_{2}$ van der Waals heterostructure via thickness variation. ACS Nano, 2019, 13: 4478-4485

$139 \mathrm{Lv} \mathrm{Q}$, Yan F, Mori N, et al. Interlayer band-to-band tunneling and negative differential resistance in van der Waals BP/InSe fieldeffect transistors. Adv Funct Mater, 2020, 30: 1910713

140 Srivastava PK, Hassan Y, Gebredingle Y, et al. Multifunctional van der Waals broken-gap heterojunction. Small, 2019, 15: 1804885

141 Fan S, Yun SJ, Yu WJ, et al. Tailoring quantum tunneling in a vanadium-doped $\mathrm{WSe}_{2} / \mathrm{SnSe}_{2}$ heterostructure. Adv Sci, 2020, 7: 1902751

$142 \mathrm{Xu} \mathrm{J}$, Jia J, Lai S, et al. Tunneling field effect transistor integrated with black phosphorus- $\mathrm{MoS}_{2}$ junction and ion gel dielectric. Appl Phys Lett, 2017, 110: 033103

143 Oliva N, Backman J, Capua L, et al. $\mathrm{WSe}_{2} / \mathrm{SnSe}_{2}$ vdW heterojunction tunnel FET with subthermionic characteristic and MOSFET co-integrated on same WSe $e_{2}$ flake. npj 2D Mater Appl,
2020, 4: 5

144 Balaji Y, Smets Q, Śzabo Á, et al. $\mathrm{MoS}_{2} / \mathrm{MoTe}_{2}$ heterostructure tunnel FETs using gated schottky contacts. Adv Funct Mater, 2020, 30: 1905970

145 Kim S, Myeong G, Shin W, et al. Thickness-controlled black phosphorus tunnel field-effect transistor for low-power switches. Nat Nanotechnol, 2020, 15: 203-206

146 Kim S, Myeong G, Park J, et al. Monolayer hexagonal boron nitride tunnel barrier contact for low-power black phosphorus heterojunction tunnel field-effect transistors. Nano Lett, 2020, 20: 3963-3969

147 Cheng L, Zhang C, Liu Y. Why two-dimensional semiconductors generally have low electron mobility. Phys Rev Lett, 2020, 125: 177701

148 Cao W, Kang J, Banerjee K. 2D/3D tunnel-FET: Toward green transistors and sensors. ECS Trans, 2017, 77: 185-189

149 Sarkar D, Xie X, Liu W, et al. A subthermionic tunnel field-effect transistor with an atomically thin channel. Nature, 2015, 526: 9195

150 Krishnamoorthy S, Lee EW, Lee CH, et al. High current density 2D/3D $\mathrm{MoS}_{2} / \mathrm{GaN}$ Esaki tunnel diodes. Appl Phys Lett, 2016, 109: 183505

151 Britnell L, Gorbachev RV, Geim AK, et al. Resonant tunnelling and negative differential conductance in graphene transistors. Nat Commun, 2013, 4: 1794

152 Mishchenko A, Tu JS, Cao Y, et al. Twist-controlled resonant tunnelling in graphene/boron nitride/graphene heterostructures. Nat Nanotech, 2014, 9: 808-813

153 Lin YC, Ghosh RK, Addou R, et al. Atomically thin resonant tunnel diodes built from synthetic van der Waals heterostructures. Nat Commun, 2015, 6: 7311

154 Campbell PM, Tarasov A, Joiner CA, et al. Enhanced resonant tunneling in symmetric 2D semiconductor vertical heterostructure transistors. ACS Nano, 2015, 9: 5000-5008

155 Fallahazad B, Lee K, Kang S, et al. Gate-tunable resonant tunneling in double bilayer graphene heterostructures. Nano Lett, 2015, 15: 428-433

156 Kang S, Fallahazad B, Lee K, et al. Bilayer graphene-hexagonal boron nitride heterostructure negative differential resistance interlayer tunnel FET. IEEE Electron Device Lett, 2015, 36: 405-407

157 Burg GW, Prasad N, Fallahazad B, et al. Coherent interlayer tunneling and negative differential resistance with high current density in double bilayer graphene-WSe $\mathrm{W}_{2}$ heterostructures. Nano Lett, 2017, 17: 3919-3925

158 Kim K, Prasad N, Movva HCP, et al. Spin-conserving resonant tunneling in twist-controlled $\mathrm{WSe}_{2}-\mathrm{hBN}-\mathrm{WSe}_{2}$ heterostructures. Nano Lett, 2018, 18: 5967-5973

159 Zheng S, Jo S, Kang K, et al. Resonant tunneling spectroscopy to probe the giant Stark effect in atomically thin materials. Adv Mater, 2020, 32: 1906942

160 Zheng S, Zhao M, Sun L, et al. Classical and quantum phases in hexagonal boron nitride-combined van der Waals heterostructures. InfoMat, 2021, 3: 252-270

161 Sup Choi M, Lee GH, Yu YJ, et al. Controlled charge trapping by molybdenum disulphide and graphene in ultrathin heterostructured memory devices. Nat Commun, 2013, 4: 1624

162 Bertolazzi S, Krasnozhon D, Kis A. Nonvolatile memory cells based on $\mathrm{MoS}_{2} /$ graphene heterostructures. ACS Nano, 2013, 7: 3246-3252

163 Wang J, Zou X, Xiao X, et al. Floating gate memory-based 
monolayer $\mathrm{MoS}_{2}$ transistor with metal nanocrystals embedded in the gate dielectrics. Small, 2015, 11: 208-213

164 Li D, Chen M, Sun Z, et al. Two-dimensional non-volatile programmable p-n junctions. Nat Nanotech, 2017, 12: 901-906

165 Liu C, Yan X, Song X, et al. A semi-floating gate memory based on van der Waals heterostructures for quasi-non-volatile applications. Nat Nanotech, 2018, 13: 404-410

166 Li D, Wang X, Zhang Q, et al. Nonvolatile floating-gate memories based on stacked black phosphorus-boron nitride- $\mathrm{MoS}_{2}$ heterostructures. Adv Funct Mater, 2015, 25: 7360-7365

167 Vu QA, Shin YS, Kim YR, et al. Two-terminal floating-gate memory with van der Waals heterostructures for ultrahigh on/off ratio. Nat Commun, 2016, 7: 12725

168 Akinwande D, Petrone N, Hone J. Two-dimensional flexible nanoelectronics. Nat Commun, 2014, 5: 5678

169 Gao L. Flexible device applications of 2D semiconductors. Small, 2017, 13: 1603994

170 Vu QA, Kim H, Nguyen VL, et al. A high-on/off-ratio floatinggate memristor array on a flexible substrate via CVD-grown large-area 2D layer stacking. Adv Mater, 2017, 29: 1703363

171 Paul T, Ahmed T, Kanhaiya Tiwari K, et al. A high-performance $\mathrm{MoS}_{2}$ synaptic device with floating gate engineering for neuromorphic computing. 2D Mater, 2019, 6: 045008

172 Mak KF, Lee C, Hone J, et al. Atomically thin $\mathrm{MoS}_{2}$ : A new directgap semiconductor. Phys Rev Lett, 2010, 105: 136805

173 Huang B, Clark G, Navarro-Moratalla E, et al. Layer-dependent ferromagnetism in a van der Waals crystal down to the monolayer limit. Nature, 2017, 546: 270-273

174 Pei J, Yang J, Yildirim T, et al. Many-body complexes in 2D semiconductors. Adv Mater, 2019, 31: 1706945

175 Ross JS, Klement P, Jones AM, et al. Electrically tunable excitonic light-emitting diodes based on monolayer $\mathrm{WSe}_{2} \mathrm{p}-\mathrm{n}$ junctions. Nat Nanotech, 2014, 9: 268-272

176 Withers F, Del Pozo-Zamudio O, Mishchenko A, et al. Lightemitting diodes by band-structure engineering in van der Waals heterostructures. Nat Mater, 2015, 14: 301-306

177 Withers F, Del Pozo-Zamudio O, Schwarz S, et al. WSe ${ }_{2}$ lightemitting tunneling transistors with enhanced brightness at room temperature. Nano Lett, 2015, 15: 8223-8228

178 Rao G, Wang X, Wang Y, et al. Two-dimensional heterostructure promoted infrared photodetection devices. InfoMat, 2019, 1: 272288

179 Koppens FHL, Mueller T, Avouris P, et al. Photodetectors based on graphene, other two-dimensional materials and hybrid systems. Nat Nanotech, 2014, 9: 780-793

180 Dong T, Simões J, Yang Z. Flexible photodetector based on 2D materials: Processing, architectures, and applications. Adv Mater Interfaces, 2020, 7: 1901657

181 Srivastava PK, Hassan Y, Gebredingle Y, et al. van der Waals broken-gap $\mathrm{p}$-n heterojunction tunnel diode based on black phosphorus and rhenium disulfide. ACS Appl Mater Interfaces, 2019, 11: 8266-8275

182 Lee J, Duong NT, Bang S, et al. Modulation of junction modes in $\mathrm{SnSe}_{2} / \mathrm{MoTe}_{2}$ broken-gap van der Waals heterostructure for multifunctional devices. Nano Lett, 2020, 20: 2370-2377

183 Zhou X, Hu X, Zhou S, et al. Tunneling diode based on $\mathrm{WSe}_{2} /$ $\mathrm{SnS}_{2}$ heterostructure incorporating high detectivity and responsivity. Adv Mater, 2018, 30: 1703286

184 Luong DH, Lee HS, Neupane GP, et al. Tunneling photocurrent assisted by interlayer excitons in staggered van der Waals hetero- bilayers. Adv Mater, 2017, 29: 1701512

$185 \mathrm{Yu}$ WJ, Vu QA, Oh H, et al. Unusually efficient photocurrent extraction in monolayer van der Waals heterostructure by tunnelling through discretized barriers. Nat Commun, 2016, 7: 13278

186 Long M, Liu E, Wang P, et al. Broadband photovoltaic detectors based on an atomically thin heterostructure. Nano Lett, 2016, 16: 2254-2259

187 Vu QA, Lee JH, Nguyen VL, et al. Tuning carrier tunneling in van der Waals heterostructures for ultrahigh detectivity. Nano Lett, 2017, 17: 453-459

188 Tao L, Chen Z, Li X, et al. Hybrid graphene tunneling photoconductor with interface engineering towards fast photoresponse and high responsivity. npj 2D Mater Appl, 2017, 1: 19

189 Britnell L, Ribeiro RM, Eckmann A, et al. Strong light-matter interactions in heterostructures of atomically thin films. Science, 2013, 340: 1311-1314

190 Li Y, Chernikov A, Zhang X, et al. Measurement of the optical dielectric function of monolayer transition-metal dichalcogenides: $\mathrm{MoS}_{2}, \mathrm{MoSe}_{2}, \mathrm{WS}_{2}$, and $\mathrm{WSe}_{2}$. Phys Rev B, 2014, 90: 205422

191 Schneider C, Glazov MM, Korn T, et al. Two-dimensional semiconductors in the regime of strong light-matter coupling. Nat Commun, 2018, 9: 2695

192 Cai Z, Cao M, Jin Z, et al. Large photoelectric-gating effect of two-dimensional van-der-Waals organic/tungsten diselenide heterointerface. npj 2D Mater Appl, 2018, 2: 21

193 Tran MD, Kim JH, Kim H, et al. Role of hole trap sites in $\mathrm{MoS}_{2}$ for inconsistency in optical and electrical phenomena. ACS Appl Mater Interfaces, 2018, 10: 10580-10586

194 Vega-Mayoral V, Borzda T, Vella D, et al. Charge trapping and coalescence dynamics in few layer $\mathrm{MoS}_{2}$. 2D Mater, 2017, 5: 015011

195 Lei S, Wen F, Li B, et al. Optoelectronic memory using twodimensional materials. Nano Lett, 2015, 15: 259-265

196 Lee J, Pak S, Lee YW, et al. Monolayer optical memory cells based on artificial trap-mediated charge storage and release. Nat Commun, 2017, 8: 14734

197 Roy K, Padmanabhan M, Goswami S, et al. Graphene-MoS 2 hybrid structures for multifunctional photoresponsive memory devices. Nat Nanotech, 2013, 8: 826-830

198 Tran MD, Kim H, Kim JS, et al. Two-terminal multibit optical memory via van der Waals heterostructure. Adv Mater, 2018, 31: 1807075

199 Liu Y, Zeng C, Zhong J, et al. Spintronics in two-dimensional materials. Nano-Micro Lett, 2020, 12: 93

200 Wang Z, Gutiérrez-Lezama I, Ubrig N, et al. Very large tunneling magnetoresistance in layered magnetic semiconductor $\mathrm{CrI}_{3}$. Nat Commun, 2018, 9: 2516

201 Ghazaryan D, Greenaway MT, Wang Z, et al. Magnon-assisted tunnelling in van der Waals heterostructures based on $\mathrm{CrBr}_{3}$. Nat Electron, 2018, 1: 344-349

202 Guo K, Deng B, Liu Z, et al. Layer dependence of stacking order in nonencapsulated few-layer $\mathrm{CrI}_{3}$. Sci China Mater, 2020, 63: 413-420

203 Long G, Henck H, Gibertini M, et al. Persistence of magnetism in atomically thin $\mathrm{MnPS}_{3}$ crystals. Nano Lett, 2020, 20: 2452-2459

204 Luong DH, Phan TL, Ghimire G, et al. Revealing antiferromagnetic transition of van der Waals $\mathrm{MnPS}_{3}$ via vertical tunneling electrical resistance measurement. APL Mater, 2019, 7: 081102

205 Klein DR, MacNeill D, Lado JL, et al. Probing magnetism in 2D 
van der Waals crystalline insulators via electron tunneling. Science, 2018, 360: 1218-1222

206 Kim HH, Yang B, Patel T, et al. One million percent tunnel magnetoresistance in a magnetic van der Waals heterostructure. Nano Lett, 2018, 18: 4885-4890

207 Song T, Cai X, Tu MWY, et al. Giant tunneling magnetoresistance in spin-filter van der Waals heterostructures. Science, 2018, 360: 1214-1218

208 Cardoso C, Soriano D, García-Martínez NA, et al. van der Waals spin valves. Phys Rev Lett, 2018, 121: 67701

209 Fei Z, Huang B, Malinowski P, et al. Two-dimensional itinerant ferromagnetism in atomically thin $\mathrm{Fe}_{3} \mathrm{GeTe}_{2}$. Nat Mater, 2018, 17: 778-782

210 Deng Y, Yu Y, Song Y, et al. Gate-tunable room-temperature ferromagnetism in two-dimensional $\mathrm{Fe}_{3} \mathrm{GeTe}_{2}$. Nature, 2018, 563: 94-99

211 Wang Z, Sapkota D, Taniguchi $\mathrm{T}$, et al. Tunneling spin valves based on $\mathrm{Fe}_{3} \mathrm{GeTe}_{2} / \mathrm{hBN} / \mathrm{Fe}_{3} \mathrm{GeTe}_{2}$ van der Waals heterostructures. Nano Lett, 2018, 18: 4303-4308

212 Dankert A, Pashaei P, Kamalakar MV, et al. Spin-polarized tunneling through chemical vapor deposited multilayer molybdenum disulfide. ACS Nano, 2017, 11: 6389-6395

213 Zhou J, Qiao J, Duan CG, et al. Large tunneling magnetoresistance in $\mathrm{VSe}_{2} / \mathrm{MoS}_{2}$ magnetic tunnel junction. ACS Appl Mater Interfaces, 2019, 11: 17647-17653

214 Yun SJ, Duong DL, Ha DM, et al. Ferromagnetic order at room temperature in monolayer $\mathrm{WSe}_{2}$ semiconductor via vanadium dopant. Adv Sci, 2020, 7: 1903076

215 Liao W, Huang Y, Wang H, et al. van der Waals heterostructures for optoelectronics: Progress and prospects. Appl Mater Today, 2019, 16: 435-455

216 Sang DK, Wen B, Gao S, et al. Electronic and optical properties of two-dimensional tellurene: From first-principles calculations. Nanomaterials, 2019, 9: 1075

217 Zhang Y, Lim CK, Dai Z, et al. Photonics and optoelectronics using nano-structured hybrid perovskite media and their optical cavities. Phys Rep, 2019, 795: 1-51

218 Fan S, Vu QA, Tran MD, et al. Transfer assembly for two-dimensional van der Waals heterostructures. 2D Mater, 2020, 7: 022005

219 Frisenda R, Navarro-Moratalla E, Gant P, et al. Recent progress in the assembly of nanodevices and van der Waals heterostructures by deterministic placement of 2D materials. Chem Soc Rev, 2018, 47: $53-68$

220 Kretinin AV, Cao Y, Tu JS, et al. Electronic properties of graphene encapsulated with different two-dimensional atomic crystals. Nano Lett, 2014, 14: 3270-3276

221 Haigh SJ, Gholinia A, Jalil R, et al. Cross-sectional imaging of individual layers and buried interfaces of graphene-based heterostructures and superlattices. Nat Mater, 2012, 11: 764-767

Acknowledgements This work was supported by China Postdoctoral Science Foundation (2020TQ0199 and 2020M682880), the Science and Technology Innovation Commission of Shenzhen (JCYJ20180305125345378), Guangdong Basic and Applied Basic Research Foundation (2020B1515020051), and the National Natural Science Foundation of China (51702219 and 61975134).

Author contributions Fan $\mathrm{S}$ was responsible for designing the writing framework and full-text writing. Cao R, Wang L, and Gao S were re- sponsible for the literature search and organization of the third section. Zhang $\mathrm{Y}, \mathrm{Yu} \mathrm{X}$, and Zhang $\mathrm{H}$ were responsible for the review of the full text.

Conflict of interest The authors declare that they have no conflict of interest.

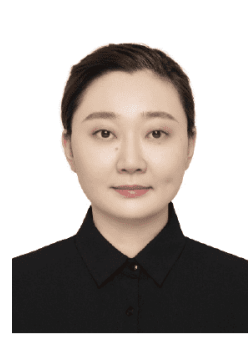

Sidi Fan received her $\mathrm{PhD}$ degree from Sungkyunkwan University, Korea. Currently, she works as a postdoctoral researcher at the International Collaborative Laboratory of 2D Materials for Optoelectronics Science and Technology of Ministry of Education, Institute of Microscale Optoelectronics, Shenzhen University, China. Her research interests focus on 2D materials and their applications in the fields of electrical and optoelectrical devices.

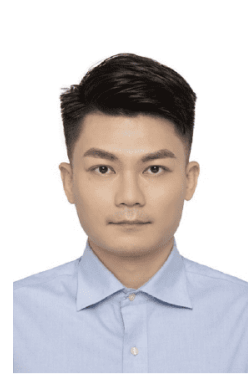

Xiang Yu received his $\mathrm{PhD}$ degree from the University of Lille, France. Currently, he works as a postdoctoral researcher at the International Collaborative Laboratory of 2D Materials for the Optoelectronics Science and Technology of Ministry of Education, Institute of Microscale Optoelectronics, Shenzhen University, China. His research interests focus on the applications of $2 \mathrm{D}$ materials in photoelectrochemistry and photocatalysis.

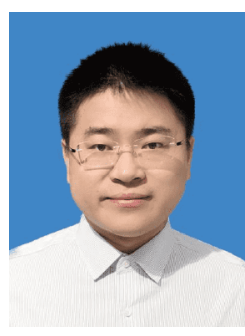

Han Zhang is a full Professor and Director of Shenzhen Engineering Laboratory of phosphorene and optoelectronics, Shenzhen University. $\mathrm{He}$ is an expert on low-dimensional optoelectronic devices and applications. Most of his publications are correlated to photonic and optoelectronic applications of 2D nanomaterials, especially graphene, TMDs, and BP.

\section{二维范德瓦尔斯异质结以及器件中的量子隧穿}

樊思迪, 曹点, 王路得, 高姗, 张豫鹏, 座翔", 张晗 ${ }^{*}$

摘要 基于能带结构的量子隧穿技术在电学、光电学、磁学等领 域有着广泛的应用. 它主要依赖于层-层的设计和构建, 是一个涵盖 了材料科学和创新技术的跨学科研究领域. 自从二维层状材料被 发现以来, 基于二维范德华异质结构的隧道器件作为下一代器件 得到了广泛的研究. 二维材料具有原子层级的厚度, 表面光滑且没 有悬挂键. 基于这些特性, 采用二维材料构建的范德瓦尔斯异质结 被认为具有优于传统 $\mathrm{Si工}$ 工艺的隧道性能, 还可获得小尺寸器件. 本 文从机理与应用两个角度对二维范德瓦尔斯异质结中的量子隧穿 现象进行了综述. 此外, 分析了二维隧道器件面临的可能性和挑战, 同时提出了对未来开发应用的展望. 二维隧道器件的技术研究和 性能控制尚处于起步阶段, 但在实现低功耗、高速以及高频功能 方面已经成为有竞争力的候选器件. 